
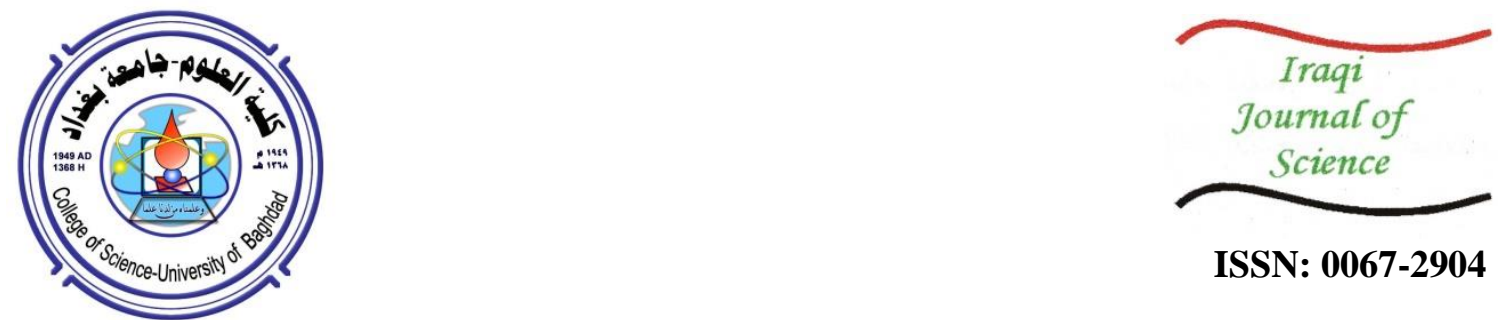

ISSN: 0067-2904

\title{
Dynamical Behavior of an eco-epidemiological Model involving Disease in predator and stage structure in prey
}

\author{
Lina Shihab Ahmed, Hassan Fadhil AL- Husseiny* \\ Department of Mathematics, College of Science, Baghdad University, Baghdad, Iraq
}

\begin{abstract}
An eco-epidemic model is proposed in this paper. It is assumed that there is a stage structure in prey and disease in predator. Existence, uniqueness and boundedness of the solution for the system are studied. The existence of each possible steady state points is discussed. The local condition for stability near each steady state point is investigated. Finally, global dynamics of the proposed model is studied numerically.
\end{abstract}

Keywords: Prey-predator model, stability, stage-structure, Disease.
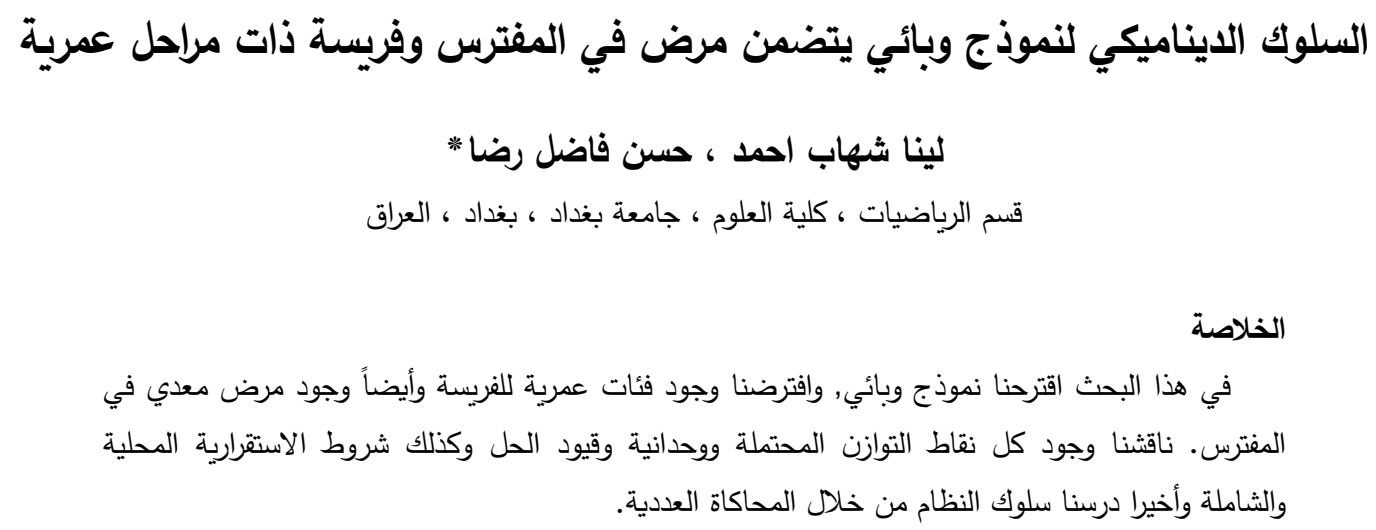

\section{Introduction}

There are many factors that affect each of prey and predator, for example, pollution of the environment, and lack of food, predation, fishing and others. In addition to the factors heir important factor is the spread of infectious diseases between the prey alone, predator, or both. Therefore, the back of a great interest by researchers to study the effect of the spread of diseases, and this type for modeling is called eco-epidemiological, such as in 1986 Anderson and May [1] were the first who merged between it, ecology and epidemic systems, they created a prey-predator model with diseases. And there are researchers proposed and studied different prey-predator models with disease spread in prey population [2-5]. As well as, there are many papers about prey-predator model with disease for example, Bairagi et.al [6] studied prey predator model with harvest and disease. Chakraborty et al. [7] studied a ratio-dependent eco epidemic model with prey harvesting and they assumed that both the susceptible and infected prey are subjected to combine harvesting. Upadhyay and Roy [8] proposed an eco-epidemic model with simple law of mass action and modified functional response in [9]. In this work, we suggested idea eco-epidemic model describing prey-predator model with epidemic disease in the prey and involving top predator. 


\section{The mathematical Formulation:}

In this section, we, the food web model consists of two compartments of predator (susceptible and infected) and a stage-structure prey in which the prey species growth logistical without of predation, while the predator decay exponentially in the absence of prey species.

It is assumed that the prey population separate into two compartments: $\mathrm{N}_{1}(\mathrm{~T})$ which represent the density of immature prey population at time $\mathrm{T}$, and $\mathrm{N}_{2}(\mathrm{~T})$ that denotes to the density of mature prey population at time T. Further the density of the susceptible predator at time $T$ is denoted by $\mathrm{N}_{3}(\mathrm{~T})$, while $\mathrm{N}_{4}(\mathrm{~T})$ represents the density of infected predator population at time $\mathrm{T}$. Now in order to formulate the dynamics of such system, the following hypotheses are adopted:

1. The immature prey depends completely in its feeding on the mature prey that grows logistically with intrinsic growth rate $\mathrm{r}>0$ and carrying capacity $\mathrm{k}>0$. The immature prey individual grow up and become mature prey with growth up rate $a_{1}>0$. However the mature prey facing death with natural death rate $\mathrm{d}_{1}>0$.

2. There is a kind of protection for the two stages of prey species from facing predation by the susceptible predator with refuge rate constant $\mathrm{m}_{1}, \mathrm{~m}_{2} \in(0,1)$ respectively.

3. The susceptible predator consumed the immature prey individuals according to Holling type-II functional response with predation rate $\mathrm{a}_{2}>0$. and half saturation constant $\mathrm{b}>0$. And consumed the mature prey individuals according to Holling type-II functional response with predation rate $\mathrm{a}_{3}>0$ and contribute of portion of such food with conversion rate $0<\mathrm{e}<1$. Moreover, the infected predator consumed the immature prey individuals according to lotka-volltera type of functional response with predation rate $\mathrm{c}>0, \mathrm{c}_{1}$ represent the disease transmission from susceptible predator to infected predator and contributes a portion of such food with conversion rate $0<\mathrm{e}_{1}<1$.

4. Finally, in the absence of food the susceptible predator. Facing death with natural death rate $d_{2}>0$ but the infected predator facing death due to disease and natural death rate $d_{3}>0$.

From above assumptions the system can be formulated mathematically with the following set of differential equations:

$$
\begin{aligned}
& \frac{d N_{1}}{d t}=r N_{2}\left(1-\frac{N_{2}}{k}\right)-a_{1} N_{1}-\frac{a_{2}\left(1-m_{1}\right) N_{1} N_{3}}{b+\left(1-m_{1}\right) N_{1}+\left(1-m_{2}\right) N_{2}} \\
& \frac{d N_{2}}{d t}=a_{1} N_{1}-\frac{a_{3}\left(1-m_{2}\right) N_{2} N_{3}}{b+\left(1-m_{1}\right) N_{1}+\left(1-m_{2}\right) N_{2}} \\
& -d_{1} N_{2} \\
& \frac{d N_{3}}{d t}=\frac{e\left[a_{2}\left(1-m_{1}\right) N_{1}+a_{3}\left(1-m_{2}\right) N_{2}\right]}{b+\left(1-m_{1}\right) N_{1}+\left(1-m_{2}\right) N_{2}} N_{3}-c_{1} N_{3} N_{4} \\
& \frac{d N_{4}}{d t} \\
& -d_{2} N_{3} \\
& =c_{1} N_{3} N_{4}+e_{1} c\left(1-m_{1}\right) N_{1} N_{4} \\
& -d_{3} N_{1}
\end{aligned}
$$

Now, by simplifying the model (1), the number of parameters is reduced by using the following dimensionless variables and parameters:

$$
\begin{array}{llll}
t=r T, & u_{1}=\frac{a_{1}}{r}, & u_{2}=\frac{s}{r}, & u_{3}=\frac{b}{k}, \quad u_{4}=\frac{a_{3}}{r}, \\
u_{5}=\frac{d_{1}}{r}, & u_{6}=\frac{c_{1}}{c}, & u_{7}=\frac{d_{2}}{r}, & u_{8}=\frac{c_{1} k}{r}, \quad u_{9}=\frac{e c k}{r}, \\
u_{10}=\frac{d_{3}}{r}, & x_{1}=\frac{N_{1}}{k}, & x_{2}=\frac{N_{2}}{k}, & x_{3}=\frac{N_{3}}{k}, \quad x_{4}=\frac{c N_{4}}{r}
\end{array}
$$

Accordingly, the dimensionless of system (1) becomes

$$
\begin{gathered}
\frac{d x_{1}}{d t}=x_{2}\left(1-x_{2}\right)-u_{1} x_{1}-u_{2}\left(1-m_{1}\right) x_{3} \frac{x_{1}}{u_{3}+\left(1-m_{1}\right) x_{1}+\left(1-m_{2}\right) x_{2}}-\left(1-m_{1}\right) x_{1} x_{4} \\
=f_{1}\left(x_{1}, x_{2}, x_{3}, x_{4}\right)
\end{gathered}
$$




$$
\begin{aligned}
& \frac{d x_{2}}{d t}=u_{1} x_{1}-\frac{u_{4}\left(1-m_{2}\right) x_{2} x_{3}}{u_{3}+\left(1-m_{1}\right) x_{1}+\left(1-m_{2}\right) x_{2}}-u_{5} x_{2} \\
& =f_{2}\left(x_{1}, x_{2}, x_{3}, x_{4}\right) \\
& \frac{d x_{3}}{d t}=x_{3}\left[e \frac{u_{2}\left(1-m_{1}\right) x_{1}+u_{4}\left(1-m_{2}\right) x_{2}}{u_{3}+\left(1-m_{1}\right) x_{1}+\left(1-m_{2}\right) x_{2}}-u_{6} x_{4}-u_{7}\right] \\
& =f_{3}\left(x_{1}, x_{2}, x_{3}, x_{4}\right) \\
& \frac{d x_{4}}{d t}=x_{4}\left[u_{8} x_{3}+\left(1-m_{1}\right) u_{9} x_{1}-u_{10}\right] \\
& =f_{4}\left(x_{1}, x_{2}, x_{3}, x_{4}\right)
\end{aligned}
$$

Clearly, the equations of system (2) are continuous and have continuous partial derivatives on the following positive 4 th dim.space:

$$
R_{+}^{4}=\left\{\left(x_{1}, x_{2}, x_{3}, x_{4}\right) \in R^{4}: x_{1}(0) \geq 0, x_{2}(0) \geq 0, \quad x_{3}(0) \geq 0, x_{4}(0) \geq 0\right\} .
$$

Therefore, these equations are Lipschizian on $\mathrm{R}_{+}^{4}$, and hence the solution of system (2) exists and unique. Furthermore, each of the solutions of system (2) with positive initial condition is bounded as shown in the following.

Theorem (1): Each of the solutions of system (2) which are initiated in $\mathrm{R}_{+}^{4}$ arebounded.

Proof: Let $\left(\mathrm{x}_{1}(\mathrm{t}), \mathrm{x}_{2}(\mathrm{t}), \mathrm{x}_{3}(\mathrm{t}), \mathrm{x}_{4}(\mathrm{t})\right)$ be a solution of system (2) with positive initial condition $\left(\mathrm{x}_{1}, \mathrm{x}_{2}, \mathrm{x}_{3}, \mathrm{x}_{4}\right) \in \mathrm{R}_{+}^{4}$.

Now define the function $M(t)=x_{1}(t)+x_{2}(t)+x_{3}(t)+x_{4}(t)$ and then taken the time derivative of $M(t)$ along the solution of system (2).

$$
\begin{aligned}
\frac{d M}{d t}=\left(1-u_{5}\right) & x_{2}-(1-e) \frac{u_{2}\left(1-m_{1}\right) x_{1} x_{3}}{u_{3}+\left(1-m_{1}\right) x_{1}+\left(1-m_{2}\right) x_{2}}-\left(1-u_{9}\right)\left(1-m_{1}\right) x_{1} x_{4} \\
& -(1-e) \frac{u_{4}\left(1-m_{2}\right) x_{2} x_{3}}{u_{3}+\left(1-m_{1}\right) x_{1}+\left(1-m_{2}\right) x_{2}}-\left(u_{6}-u_{8}\right) x_{3} x_{4}-u_{7} x_{3}-u_{10} x_{4}
\end{aligned}
$$

So, due to the fact that the conversion rate constant from prey population to predator population cannot exceeding the maximum predation rate constant from predator population to prey population, hence from the biological point of view, always $\mathrm{u}_{5}<1$, e $<1, \mathrm{u}_{9}<1, \mathrm{u}_{6}<\mathrm{u}_{8}$ we get:

$$
\begin{gathered}
\frac{d M}{d t}=x_{1}+x_{2}-\left(x_{1}+x_{2}+u_{7} x_{3}\right. \\
\left.+u_{10} x_{4}\right) \\
\frac{d M}{d t} \leq x_{1}+x_{2}-\mu\left(x_{1}+x_{2}+x_{3}\right. \\
\left.+x_{4}\right)
\end{gathered}
$$

Where $\mu=\min \left\{1, \mathrm{u}_{7}, \mathrm{u}_{10}\right\}$

Since $\mathrm{x}_{1}+\mathrm{x}_{2}=\mathrm{N}$ represents prey specie which is growth logistically with carrying capacity(1), hence $\mathrm{N} \leq 1$

So that,

$$
\frac{\mathrm{dM}}{\mathrm{dt}} \leq 1-\mu \mathrm{M} \text {. }
$$

Now, solve the differential equation with initial value $M(0)=M_{0}$ we get:

$$
M(t) \leq \frac{1}{\mu}+\left(M_{0}-\frac{1}{\mu}\right) e^{-\mu t}
$$

Then,

$$
\lim _{\mathrm{t} \rightarrow \infty} M(\mathrm{t}) \leq \lim _{\mathrm{t} \rightarrow \infty} \frac{1}{\mu}+\lim _{\mathrm{t} \rightarrow \infty}\left(\mathrm{M}_{0}-\frac{1}{\mu}\right) \mathrm{e}^{-\mu \mathrm{t}} .
$$

So

$$
\mathrm{M}(\mathrm{t}) \leq \frac{1}{\mu}, \forall \mathrm{t}>0 .
$$

Then each the solution of system (2 )uniformly bounded. 


\section{Existence of the steady state points}

In this part, the existence of all possible steady state points of system (2) is discussed. It is observed that, system (2) has only four steady state points, which are mentioned in the following:

- $\quad$ The steady state point $E_{0}=(0,0,0,0)$, which is known as the varieshing point and is always exists.

- $\quad$ The two species steady state point $E_{1}=\left(\bar{x}_{1}, \bar{x}_{2}, 0,0\right)$ where:

$$
\left.\begin{array}{l}
\overline{\mathrm{x}}_{1}=\frac{\left(1-\mathrm{u}_{5}\right) \mathrm{u}_{5}}{\mathrm{u}_{1}}>0 \\
\overline{\mathrm{x}}_{2}=\left(1-\mathrm{u}_{5}\right)>0
\end{array}\right\}
$$

Exists under the following condition

$\mathrm{u}_{5}<1$.

- $\quad$ The steady state point $\mathrm{E}_{2}=\left(\hat{\mathrm{x}}_{1}, \hat{\mathrm{x}}_{2}, \hat{\mathrm{x}}_{3}, 0\right)$

$\hat{\mathrm{x}}_{1}$

$=\frac{u_{3} u_{7}+\left[u_{7}-e u_{5}\right]\left(1-m_{2}\right) x_{2}}{\left[e u_{2}-u_{7}\right]\left(1-m_{1}\right)}$

From the second equation of system (2) we have

$$
\begin{gathered}
u_{1} u_{3} x_{1}+u_{1}\left(1-m_{1}\right) x_{1}^{2}+u_{1}\left(1-m_{2}\right) x_{1} x_{2}-u_{4}\left(1-m_{2}\right) x_{2} x_{3}-u_{5} u_{3} x_{2}-u_{5}\left(1-m_{1}\right) x_{1} x_{2} \\
-u_{5}\left(1-m_{2}\right) x_{2}^{2}=0
\end{gathered}
$$

By substituting equation (4a) in equation (4b) we get

$$
\begin{aligned}
& {\left[u_{1}\left(u_{7}-e u_{5}\right)\left(e u_{2}-u_{7}\right)\left(1-m_{1}\right)\left(1-m_{2}\right)^{2}+u_{1}\left(u_{7}-e u_{5}\right)^{2}\left(1-m_{1}\right)\left(1-m_{2}\right)^{2}\right.} \\
& \quad-u_{5}\left(u_{7}-e u_{5}\right)\left(e u_{2}-u_{7}\right)\left(1-m_{1}\right)^{2}\left(1-m_{2}\right) \\
& \quad-u_{5}\left(1-m_{2}\right)\left(\left(e u_{2}-u_{7}\right)\left(1-m_{1}\right)^{2}\right] x_{2}^{2} \\
& +\left[u_{1} u_{3}\left(u_{7}-e u_{5}\right)\left(e u_{2}-u_{7}\right)\left(1-m_{1}\right)\left(1-m_{2}\right)\right. \\
& \quad+2 u_{1} u_{3} u_{7}\left(u_{7}-e u_{5}\right)\left(1-m_{1}\right)\left(1-m_{2}\right)+u_{1} u_{3} u_{7}\left(e u_{2}-u_{7}\right)\left(1-m_{1}\right)\left(1-m_{2}\right) \\
& \left.\quad-u_{3} u_{5} u_{7}\left(e u_{2}-u_{7}\right)\left(1-m_{1}\right)^{2}\right] x_{2}-u_{4}\left(1-m_{2}\right)\left(\left(e u_{2}-u_{7}\right)\left(1-m_{1}\right)\right)^{2} x_{2} x_{3} \\
& \quad+\left[u_{1} u_{3}^{2} u_{7}\left(\left(e u_{2}-u_{7}\right)\left(1-m_{1}\right)\right)+u_{1} u_{3}^{2} u_{7}^{2}\left(1-m_{1}\right)\right]=0=f_{1}\left(x_{2}, x_{3}\right) \\
& x_{2}-x_{2}^{2}-u_{1} x_{1}-u_{2}\left(1-m_{1}\right) \frac{x_{1} x_{3}}{u_{3}+\left(1-m_{1}\right) x_{1}+\left(1-m_{2}\right) x_{2}} \\
& =0
\end{aligned}
$$

Also by substituting equation (4a) in equation $(4 \mathrm{c})$ we get:

$$
\begin{aligned}
{\left[-\left(1-m_{2}\right)((\right.} & \left.\left.\left(e u_{2}-u_{7}\right)\left(1-m_{1}\right)\right)^{2}-\left(1-m_{1}\right)\left(\left(u_{7}-e u_{5}\right)\left(1-m_{2}\right)\right)\left(\left(e u_{2}-u_{7}\right)\left(1-m_{1}\right)\right)\right] x_{2}^{3} \\
& +\left[\left(\left(1-m_{2}\right)-u_{3}\right)\left(\left(e u_{2}-u_{7}\right)\left(1-m_{1}\right)\right)^{2}\right. \\
& +\left(\left(1-m_{1}\right)-u_{1}\left(1-m_{2}\right)\right)\left(\left(e u_{2}-u_{7}\right)\left(1-m_{1}\right)\right)\left(\left(u_{7}-e u_{5}\right)\left(1-m_{2}\right)\right) \\
& \left.-u_{3} u_{7}\left(1-m_{1}\right)\left(\left(e u_{2}-u_{7}\right)\left(1-m_{1}\right)\right)-u_{1}\left(1-m_{1}\right)\left(\left(u_{7}-e u_{5}\right)\left(1-m_{2}\right)\right)^{2}\right] x_{2}^{2} \\
& +\left[u_{3}\left(\left(e u_{2}-u_{7}\right)\left(1-m_{1}\right)\right)^{2}\right. \\
& +u_{3} u_{7}\left(\left(1-m_{1}\right)-u_{1}\left(1-m_{2}\right)\right)\left(\left(e u_{2}-u_{7}\right)\left(1-m_{1}\right)\right) \\
& -u_{1} u_{3}\left(\left(u_{7}-e u_{5}\right)\left(1-m_{2}\right)\right)\left(\left(e u_{2}-u_{7}\right)\left(1-m_{1}\right)\right) \\
& \left.-2 u_{1} u_{3} u_{7}\left(1-m_{1}\right)\left(u_{7}-e u_{5}\right)\left(1-m_{2}\right)\right] x_{2}\left[-u_{1} u_{3}^{2} u_{7}^{2}\left(1-m_{1}\right)\right]\left[-u_{2} u_{3} u_{7}(1\right. \\
& \left.\left.-m_{1}\right)\left(\left(e u_{2}-u_{7}\right)\left(1-m_{1}\right)\right)\right] x_{3}\left[-u_{2}(1\right. \\
& \left.\left.-m_{1}\right)\left(\left(u_{7}-e u_{5}\right)\left(1-m_{2}\right)\right)\left(\left(e u_{2}-u_{7}\right)\left(1-m_{1}\right)\right)\right] x_{2} x_{3}=0=f_{2}\left(x_{2}, x_{3}\right)
\end{aligned}
$$




$$
f_{2}\left(x_{2}, x_{3}\right)=b_{1} x_{2}^{3}+b_{2} x_{2}^{2}+b_{3} x_{2}+b_{4}+b_{5} x_{3}+b_{6} x_{2} x_{3}=0
$$

Where

$$
\begin{aligned}
& a_{1}=\left[u_{1}\left(u_{7}-e u_{5}\right)\left(e u_{2}-u_{7}\right)\left(1-m_{1}\right)\left(1-m_{2}\right)^{2}+u_{1}\left(u_{7}-e u_{5}\right)^{2}\left(1-m_{1}\right)\left(1-m_{2}\right)^{2}\right. \\
& -u_{5}\left(u_{7}-e u_{5}\right)\left(e u_{2}-u_{7}\right)\left(1-m_{1}\right)^{2}\left(1-m_{2}\right) \\
& \left.-u_{5}\left(1-m_{2}\right)\left(\left(e u_{2}-u_{7}\right)\left(1-m_{1}\right)\right)^{2}\right] \\
& a_{2}=\left[u_{1} u_{3}\left(u_{7}-e u_{5}\right)\left(e u_{2}-u_{7}\right)\left(1-m_{1}\right)\left(1-m_{2}\right)\right. \\
& +2 u_{1} u_{3} u_{7}\left(u_{7}-e u_{5}\right)\left(1-m_{1}\right)\left(1-m_{2}\right) \\
& +u_{1} u_{3} u_{7}\left(e u_{2}-u_{7}\right)\left(1-m_{1}\right)\left(1-m_{2}\right)-u_{3} u_{5} u_{7}\left(e u_{2}-u_{7}\right)\left(1-m_{1}\right)^{2} \\
& \left.-u_{3} u_{5}\left(\left(e u_{2}-u_{7}\right)\left(1-m_{1}\right)\right)^{2}\right] \\
& a_{3}=-u_{4}\left(1-m_{2}\right)\left(\left(e u_{2}-u_{7}\right)\left(1-m_{1}\right)\right)^{2} \\
& <0 \\
& a_{4}=\left[u_{1} u_{3}^{2} u_{7}\left(\left(e u_{2}-u_{7}\right)\left(1-m_{1}\right)\right)+u_{1} u_{3}^{2} u_{7}^{2}\left(1-m_{1}\right)\right] \\
& b_{1}=\left[-\left(1-m_{2}\right)\left(\left(e u_{2}-u_{7}\right)\left(1-m_{1}\right)\right)^{2}\right. \\
& \left.-\left(1-m_{1}\right)\left(\left(u_{7}-e u_{5}\right)\left(1-m_{2}\right)\right)\left(\left(e u_{2}-u_{7}\right)\left(1-m_{1}\right)\right)\right] \\
& b_{2}=\left[\left(\left(1-m_{2}\right)-u_{3}\right)\left(\left(e u_{2}-u_{7}\right)\left(1-m_{1}\right)\right)^{2}\right. \\
& +\left(\left(1-m_{1}\right)-u_{1}\left(1-m_{2}\right)\right)\left(\left(e u_{2}-u_{7}\right)\left(1-m_{1}\right)\right)\left(\left(u_{7}-e u_{5}\right)\left(1-m_{2}\right)\right) \\
& -u_{3} u_{7}\left(1-m_{1}\right)\left(\left(e u_{2}-u_{7}\right)\left(1-m_{1}\right)\right) \\
& -u_{1}\left(1-m_{1}\right)\left(\left(u_{7}-e u_{5}\right)\left(1-m_{2}\right)\right)^{2} \\
& b_{3}=\left[u_{3}\left(\left(e u_{2}-u_{7}\right)\left(1-m_{1}\right)\right)^{2}+u_{3} u_{7}\left(\left(1-m_{1}\right)-u_{1}\left(1-m_{2}\right)\right)\left(\left(e u_{2}-u_{7}\right)\left(1-m_{1}\right)\right)\right. \\
& -u_{1} u_{3}\left(\left(u_{7}-e u_{5}\right)\left(1-m_{2}\right)\right)\left(\left(e u_{2}-u_{7}\right)\left(1-m_{1}\right)\right) \\
& \left.-2 u_{1} u_{3} u_{7}\left(1-m_{1}\right)\left(u_{7}-e u_{5}\right)\left(1-m_{2}\right)\right] \\
& b_{4}=-u_{1} u_{3}^{2} u_{7}\left(\left(e u_{2}-u_{7}\right)\left(1-m_{1}\right)\right)-u_{1} u_{3}^{2} u_{7}^{2}\left(1-m_{1}\right) \\
& <0 \\
& b_{5}=-u_{2} u_{3} u_{7}\left(1-m_{1}\right)\left(\left(e u_{2}-u_{7}\right)\left(1-m_{1}\right)\right) \\
& <0 \\
& b_{6}=-u_{2}\left(1-m_{1}\right)\left(\left(u_{7}-e u_{5}\right)\left(1-m_{2}\right)\right)\left(\left(e u_{2}-u_{7}\right)\left(1-m_{1}\right)\right)
\end{aligned}
$$

Now, in order to determine the values of $\hat{\mathrm{x}}_{2}$ and $\hat{\mathrm{x}}_{3}$, consider the two isoclines $(4 \dot{\mathrm{d}})$ and $(4 \mathrm{e})$ as $\mathrm{x}_{3} \rightarrow 0$ which gives:

$$
\begin{aligned}
& \mathrm{f}_{1}\left(\mathrm{x}_{2}\right)=\mathrm{a}_{1} \mathrm{x}_{2}^{2}+\mathrm{a}_{2} \mathrm{x}_{2}+\mathrm{a}_{4}=0 \\
& \mathrm{f}_{2}\left(\mathrm{x}_{2}\right)=\mathrm{b}_{1} \mathrm{x}_{2}^{3}+\mathrm{b}_{2} \mathrm{x}_{2}^{2}+\mathrm{b}_{3} \mathrm{x}_{2}+\mathrm{b}_{4}=0
\end{aligned}
$$

Obviously equation (i) is second degree polynomial equation, while equation (i i) is a third degree polynomial equation.

Consequently eq.(i) have a positive root say $\mathrm{x}_{2 \mathrm{a}}$ provided that one of the following conditions hold:

$$
\left.\begin{array}{ll}
\mathrm{a}_{1}>0 & \text { and } \mathrm{a}_{4}<0 \\
& \text { or } \\
\mathrm{a}_{1}<0 & \text { and } \mathrm{a}_{4}>0
\end{array}\right\}
$$

However, equation (i i)has just one positive root, say $\mathrm{x}_{2 \mathrm{~b}}$, provided that one of the following conditions hold:

$$
\left.\begin{array}{ll}
b_{1}>0 & \text { and } b_{2}>0 \\
b_{1}>0 & \text { or } \\
\text { and } b_{3}<0
\end{array}\right\}
$$

Since we have $f\left(x_{2}, x_{3}\right)=0$ then the derivative with respect to $x_{2}$ becomes: 
$\frac{d x_{3}}{d x_{2}}$
$=-\frac{2 a_{1} x_{2}+a_{2}+a_{3} x_{3}}{a_{3} x_{2}}$

Note that, $\frac{d x_{3}}{d x_{2}}<0$ and hence the isoclines (4d) is Decreasing if the following condition hold:

Similarly from equation (4e), we noted:

$$
2 a_{1} x_{2}+a_{2}+a_{3} x_{3}>0
$$

$$
\frac{d x_{3}}{d x_{2}}=-\frac{3 b_{1} x_{2}^{2}+2 b_{2} x_{2}+b_{3}+b_{6} x_{3}}{b_{5}+b_{6} x_{2}}
$$

Here $\frac{d x_{3}}{d x_{2}}>0$ and hence the isoclines (4e) is increasing function iff the following condition hold:

$$
3 b_{1} x_{2}^{2}+2 b_{2} x_{2}+b_{3}+b_{6} x_{3}<0
$$

Now, if $\mathrm{x}_{2 \mathrm{~b}}<\mathrm{x}_{2 \mathrm{a}}$, we get by the above analysis, it is noted that the two isoclines (4d) and (4e) intersect at unique point $\left(\mathrm{x}_{2}, \mathrm{x}_{3}\right)$ iff the conditions $(4 \mathrm{f}),(4 \mathrm{~g}),(4 \mathrm{~h})$ and $(4 \mathrm{i})$ are satisfied, and hence the system (2) has only one positive steady state point if in addition to these conditions the following holds:

$$
\frac{\mathrm{eu}_{5}\left(1-\mathrm{m}_{2}\right) \hat{\mathrm{x}}_{2}}{\mathrm{u}_{3}+\left(1-\mathrm{m}_{2}\right) \hat{\mathrm{x}}_{2}}>\mathrm{u}_{7}>e u_{5}
$$

- Lastly, the positive (coexistence) steady state point $\mathrm{E}_{3}=\left(\breve{\mathrm{x}}_{1}, \breve{\mathrm{x}}_{2}, \mathrm{x}_{3}, \breve{\mathrm{x}}_{4}\right)$ exists if there is positive solution to the following set of equation:

$$
\begin{aligned}
& x_{2}-x_{2}^{2}-u_{1} x_{1}-u_{2}\left(1-m_{1}\right) \frac{x_{1} x_{3}}{u_{3}+\left(1-m_{1}\right) x_{1}+\left(1-m_{2}\right) x_{2}}-\left(1-m_{1}\right) x_{1} x_{4} \\
& =0 \quad \text { (5a) } \\
& u_{1} x_{1}-u_{4}\left(1-m_{2}\right) \frac{x_{2} x_{3}}{u_{3}+\left(1-m_{1}\right) x_{1}+\left(1-m_{2}\right) x_{2}}-u_{5} x_{2}=0 \\
& x_{3}\left[e \frac{u_{2}\left(1-m_{1}\right) x_{1}+u_{4}\left(1-m_{2}\right) x_{2}}{u_{3}+\left(1-m_{1}\right) x_{1}+\left(1-m_{2}\right) x_{2}}-u_{6} x_{4}-u_{7}\right]=0 \\
& x_{4}\left[u_{8} x_{3}+\left(1-m_{1}\right) u_{9} x_{1}-u_{10}\right]=0 \\
& \text { From equation }(5 d) \text { we obtain: } \\
& \breve{x}_{3}=\frac{u_{10}-\left(1-m_{1}\right) u_{9} x_{1}}{u_{8}} \\
& \breve{x}_{4}=\frac{\left(e u_{2}-u_{7}\right)\left(1-m_{1}\right) x_{1}+\left(e u_{4}-u_{7}\right)\left(1-m_{2}\right)-u_{3} u_{7}}{u_{6}\left[u_{3}+\left(1-m_{1}\right) x_{1}+\left(1-m_{2}\right) x_{2}\right]}
\end{aligned}
$$

From equation $(5 \mathrm{~b})$ we get:

$$
\begin{aligned}
u_{1} u_{8}\left(1-m_{1}\right) & x_{1}^{2}+u_{1} u_{3} u_{8} x_{1} \\
& +\left[u_{1} u_{8}\left(1-m_{2}\right)+u_{4} u_{9}\left(1-m_{1}\right)\left(1-m_{2}\right)-u_{5} u_{8}\left(1-m_{1}\right)\right] x_{1} x_{2} \\
& +\left[-u_{4} u_{10}\left(1-m_{2}\right)-u_{3} u_{5} u_{8}\right] x_{2}-u_{5} u_{8}\left(1-m_{2}\right) x_{2}^{2}=f\left(x_{1}, x_{2}\right)
\end{aligned}
$$

Substituting equations (5e) and (5f) in equation (5a) we get:

$$
\begin{aligned}
-u_{6} u_{8}\left(1-m_{2}\right) & x_{2}^{3}+\left[u_{6} u_{8}\left(1-m_{2}\right)-u_{3} u_{6} u_{8}\right] x_{2}^{2}+u_{6} u_{8} u_{3} x_{2}-u_{6} u_{8}\left(1-m_{1}\right) x_{1} x_{2}^{2} \\
+ & {\left[u_{6} u_{8}\left(1-m_{1}\right)-u_{1} u_{6} u_{8}\left(1-m_{2}\right)-u_{8}\left(e u_{4}-u_{7}\right)\left(1-m_{1}\right)\left(1-m_{2}\right)\right] x_{1} x_{2} } \\
+ & {\left[u_{2} u_{6} u_{9}\left(1-m_{1}\right)^{2}-u_{1} u_{6} u_{8}\left(1-m_{1}\right)-u_{8}\left(e u_{2}-u_{7}\right)\left(1-m_{1}\right)^{2}\right] x_{1}^{2} } \\
+ & {\left[u_{3} u_{7} u_{8}\left(1-m_{1}\right)-u_{2} u_{6} u_{10}\left(1-m_{1}\right)-u_{1} u_{3} u_{6} u_{8}\right] x_{1}=0=g\left(x_{1}, x_{2}\right) }
\end{aligned}
$$

Now, with some simplification we have:

$$
\begin{aligned}
& f\left(x_{1}, x_{2}\right)=r_{1} x_{1}^{2}+r_{2} x_{1}+r_{3} x_{1} x_{2}+r_{4} x_{2}+r_{4} x_{2}^{2}=0 \\
& g\left(x_{1}, x_{2}\right)=s_{1} x_{2}^{3}+s_{2} x_{2}^{2}+s_{3} x_{2}+s_{4} x_{1} x_{2}^{2}+s_{5} x_{1} x_{2}+s_{6} x_{1}^{2}+s_{7} x_{1}=0
\end{aligned}
$$

Where

$$
\begin{gathered}
r_{1}=u_{1} u_{8}\left(1-m_{1}\right)>0, r_{2}=u_{1} u_{3} u_{8} \\
>0 \\
r_{3}=u_{1} u_{8}\left(1-m_{2}\right)+u_{4} u_{9}\left(1-m_{1}\right)\left(1-m_{2}\right)-u_{5} u_{8}\left(1-m_{1}\right) \\
r_{4}=-u_{4} u_{10}\left(1-m_{2}\right)-u_{3} u_{5} u_{8}<0 \\
r_{5}=-u_{5} u_{8}\left(1-m_{2}\right)<0
\end{gathered}
$$




$$
\begin{aligned}
& s_{1}=-u_{6} u_{8}\left(1-m_{2}\right)<0 \\
& s_{2}=u_{6} u_{8}\left(1-m_{2}\right)-u_{3} u_{6} u_{8} \\
& s_{3}=u_{3} u_{6} u_{8}>0 \\
& s_{4}=-u_{6} u_{8}\left(1-m_{1}\right)<0 \\
& s_{5}=u_{6} u_{8}\left(1-m_{1}\right)-u_{1} u_{6} u_{8}\left(1-m_{2}\right)-u_{8}\left(e u_{4}-u_{7}\right)\left(1-m_{1}\right)\left(1-m_{2}\right) \\
& \quad s_{6}=u_{2} u_{6} u_{9}\left(1-m_{1}\right)^{2}-u_{1} u_{6} u_{8}\left(1-m_{1}\right)-u_{8}\left(e u_{2}-u_{7}\right)\left(1-m_{1}\right)^{2} \\
& s_{7}=u_{3} u_{7} u_{8}\left(1-m_{1}\right)-u_{2} u_{6} u_{10}\left(1-m_{1}\right)-u_{1} u_{3} u_{6} u_{8}
\end{aligned}
$$

Now, in order to determine the values of $\breve{x}_{1}$ and $\breve{x}_{2}$, consider the two isoclines $i$ and ii as $x_{1} \rightarrow 0$, which gives:

$$
\begin{gathered}
f\left(x_{2}\right)=r_{4} x_{2}+r_{5} x_{2}^{2}=x_{2}\left(r_{4}+r_{5} x_{2}\right)=0 \\
g\left(x_{2}\right)=s_{1} x_{2}^{3}+s_{2} x_{2}^{2}+s_{3} x_{2}=0
\end{gathered}
$$

Obviously equation (i) is second degree polynomial equation, while equation (ii) is a third degree polynomial consequently due to Descartes' rule equation (i)has two roots one of them, say $\breve{\mathrm{x}}_{2 \mathrm{n}}=$ 0 other of them say $x_{2 m}=\frac{-r_{4}}{r_{5}}<0$, However, equation (ii) has a unique positive root, say $x_{2 w}$ and from equation $(5 \mathrm{~g})$ it is easy to verify that

$\frac{d x_{1}}{d x_{2}}$

$=-\frac{r_{3} x_{1}+r_{4}+2 r_{5} x_{2}}{2 r_{1} x_{1}+r_{2}+r_{3} x_{2}}$

Hence, $\frac{\mathrm{dx}_{1}}{\mathrm{dx}_{2}}>0$ and hence the isoclines $(5 \mathrm{~g})$ is increasing function if the following condition hold:

$$
\left.\begin{array}{c}
r_{3} x_{1}+r_{4}+2 r_{5} x_{2}<0 \\
2 r_{1} x_{1}+r_{2}+r_{3} x_{2}>0 \\
O R \\
r_{3} x_{1}+r_{4}+2 r_{5} x_{2}>0 \\
2 r_{1} x_{1}+r_{2}+r_{3} x_{2}<0
\end{array}\right\}
$$

Similarly from equation ( $5 h$ ), we noted

$$
\begin{aligned}
& \frac{d x_{1}}{d x_{2}} \\
& =-\frac{3 s_{1} x_{2}^{2}+2 s_{2} x_{2}+s_{3}+2 s_{4} x_{1} x_{2}+s_{5} x_{2}}{s_{4} x_{2}^{2}+s_{5} x_{2}+2 s_{6} x_{1}+s_{7}}
\end{aligned}
$$

Note that $\frac{\mathrm{dx}_{1}}{\mathrm{dx}_{2}}<0$ and hence the isoclines $(5 \mathrm{~h})$ is decreasing iff the following condition hold:

$$
\left.\begin{array}{c}
3 s_{1} x_{2}^{2}+2 s_{2} x_{2}+s_{3}+2 s_{4} x_{1} x_{2}+s_{5} x_{2}>0 \\
s_{4} x_{2}^{2}+s_{5} x_{2}+2 s_{6} x_{1}+s_{7}>0 \\
O R \\
3 s_{1} x_{2}^{2}+2 s_{2} x_{2}+s_{3}+2 s_{4} x_{1} x_{2}+s_{5} x_{2}<0 \\
s_{4} x_{2}^{2}+s_{5} x_{2}+2 s_{6} x_{1}+s_{7}<0
\end{array}\right\}
$$

Therefore the positive equilibrium point $E_{3}$ exists uniquely provided that in addition to the above conditions the following two conditions hold

$$
\begin{gathered}
\bar{x}_{1}<\frac{u_{10}}{u_{9}\left(1-m_{1}\right)} \\
\left(e u_{2}-u_{7}\right)\left(1-m_{1}\right) x_{1}+\left(e u_{2}-u_{7}\right)\left(1-m_{2}\right) x_{2}>u_{3} u_{7}
\end{gathered}
$$

\section{The stability Conditions}

In this part, the local conditions for stability near the steady state points of system (2) is investigated. It is to verify that the Jacobian matrix of system (2), at the general point $\left(\mathrm{x}_{1}, \mathrm{x}_{2}, \mathrm{x}_{3}, \mathrm{x}_{4}\right)$

$$
\begin{gathered}
J=(\text { dig })_{4 \times 4} \quad i, j=1,2,3,4 . \\
a_{11}=-\left[u_{1}+\left(1-m_{1}\right) x_{4}\right]-\left[\frac{u_{2} u_{3}\left(1-m_{1}\right) x_{3}+u_{2}\left(1-m_{1}\right)\left(1-m_{2}\right) x_{2} x_{3}}{\left(u_{3}+\left(1-m_{1}\right) x_{1}+\left(1-m_{2}\right) x_{2}\right)^{2}}\right]
\end{gathered}
$$




$$
\begin{gathered}
a_{12}=1-2 x_{2}+\left[\frac{\left(u_{2}\left(1-m_{1}\right) x_{1} x_{3}\right)\left(1-m_{2}\right)}{\left(u_{3}+\left(1-m_{1}\right) x_{1}+\left(1-m_{2}\right) x_{2}\right)^{2}}\right] \\
a_{13}=\frac{-u_{2}\left(1-m_{1}\right) x_{1}}{u_{3}+\left(1-m_{1}\right) x_{1}+\left(1-m_{2}\right) x_{2}} \\
a_{14}=-\left(1-m_{1}\right) x_{1} \\
a_{21}=u_{1}+\frac{u_{4}\left(1-m_{1}\right)\left(1-m_{2}\right) x_{2} x_{3}}{\left(u_{3}+\left(1-m_{1}\right) x_{1}+\left(1-m_{2}\right) x_{2}\right)^{2}} \\
a_{22}=-u_{5}-\left[\frac{u_{3} u_{4}\left(1-m_{2}\right) x_{3}+u_{4}\left(1-m_{1}\right)\left(1-m_{2}\right) x_{1} x_{3}}{\left(u_{3}+\left(1-m_{1}\right) x_{1}+\left(1-m_{2}\right) x_{2}\right)^{2}}\right] \\
a_{23}=\frac{-u_{4}\left(1-m_{2}\right) x_{2}}{u_{3}+\left(1-m_{1}\right) x_{1}+\left(1-m_{2}\right) x_{2}} \quad, \quad a_{24}=0 \\
a_{31}=\frac{e u_{2} u_{3}\left(1-m_{1}\right) x_{3}+\left[u_{2}-u_{4}\right] e\left(1-m_{1}\right)\left(1-m_{2}\right) x_{2} x_{3}}{\left(u_{3}+\left(1-m_{1}\right) x_{1}+\left(1-m_{2}\right) x_{2}\right)^{2}} \\
a_{32}=\frac{e u_{3} u_{4}\left(1-m_{2}\right) x_{3}+\left[u_{4}-u_{2}\right] e\left(1-m_{1}\right)\left(1-m_{2}\right) x_{1} x_{3}}{\left(u_{3}+\left(1-m_{1}\right) x_{1}+\left(1-m_{2}\right) x_{2}\right)^{2}} \\
a_{33}=\frac{e u_{2}\left(1-m_{1}\right) x_{1}+e u_{4}\left(1-m_{2}\right) x_{2}}{u_{3}+\left(1-m_{1}\right) x_{1}+\left(1-m_{2}\right) x_{2}}-\left(u_{6} x_{4}+u_{7}\right) \\
a_{34}=-u_{6} x_{3}, a_{41}=\left(1-m_{1}\right) u_{9} x_{4}, \\
a_{42}=0 \quad, \quad a_{43}=u_{8} x_{3} \\
a_{44}=u_{8} x_{3}+u_{9}\left(1-m_{1}\right) x_{1}-u_{10}
\end{gathered}
$$

Therefore, the Jacobian matrix of system (2) at the vanishing steady state point $E_{0}$ is:

$$
J\left(E_{0}\right)=\left[\begin{array}{cccc}
-u_{1} & 1 & 0 & 0 \\
u_{1} & -u_{5} & 0 & 0 \\
0 & 0 & -u_{7} & 0 \\
0 & 0 & 0 & -u_{10}
\end{array}\right]
$$

Thus the eigenvalues of $\mathrm{J}\left(\mathrm{E}_{0}\right)$ are

Either $\lambda_{\mathrm{x}_{3}}=-\mathrm{u}_{7}<0$ and $\lambda_{\mathrm{x}_{4}}=-\mathrm{u}_{10}<0$ or $\lambda^{2}+\mathrm{B}_{1} \lambda+\mathrm{B}_{2}=0$ which gives two eigenvalues

$$
\lambda_{x_{1}, x_{2}}=\frac{-B_{1}}{2} \pm \frac{1}{2} \sqrt{B_{1}^{2}-4 B_{2}}
$$

where

0

$$
B_{1}=u_{1}+u_{5}>
$$

0

$$
B_{2}=u_{1}\left(u_{5}-1\right)<
$$

Therefore, $E_{0}$ is a saddle point.

The Jacobian matrix of system (2) at $E_{1}$ is given by

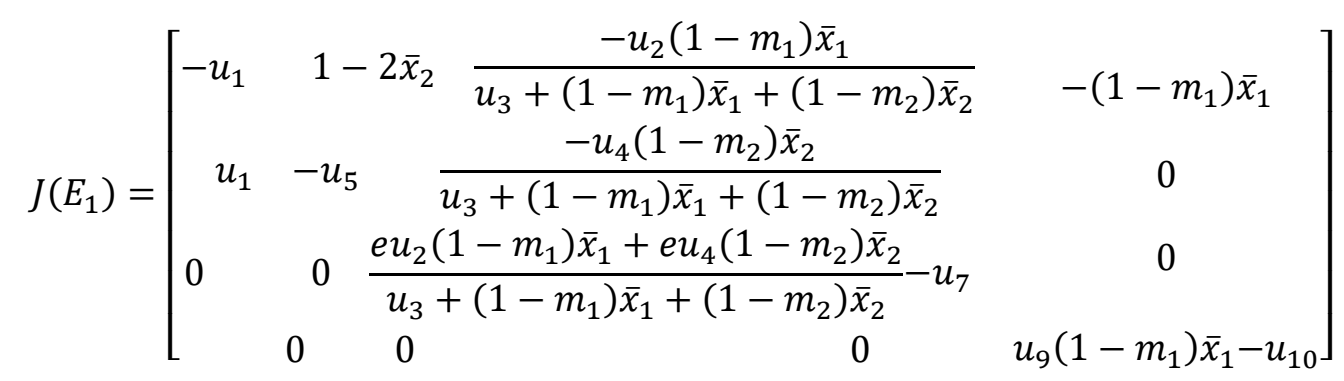

Accordingly the characteristic equation of $\left(\mathrm{E}_{1}\right)$ canbe written as: 


$$
\left[\lambda-\left(u_{9}\left(1-m_{1}\right) \bar{x}_{1}-u_{10}\right)\right]\left[\lambda-\left(\frac{e u_{2}\left(1-m_{1}\right) \bar{x}_{1}+e u_{4}\left(1-m_{2}\right) \bar{x}_{2}}{u_{3}+\left(1-m_{1}\right) \bar{x}_{1}+\left(1-m_{2}\right) \bar{x}_{2}}-u_{7}\right)\right]\left[\lambda^{2}+B_{1} \lambda+B_{2}\right]=0
$$

Where

$$
\begin{aligned}
& B_{1}=-\left[-u_{1}-u_{5}\right]=u_{1}+u_{5} \\
& B_{2}=u_{1} u_{5}-u_{1}\left(1-2 \bar{x}_{2}\right)
\end{aligned}
$$

\section{So either}

$$
\left[\lambda-\left(u_{9}\left(1-m_{1}\right) \bar{x}_{1}-u_{10}\right)\right]\left[\lambda-\left(\frac{e u_{2}\left(1-m_{1}\right) \bar{x}_{1}+e u_{4}\left(1-m_{2}\right) \bar{x}_{2}}{u_{3}+\left(1-m_{1}\right) \bar{x}_{1}+\left(1-m_{2}\right) \bar{x}_{2}}-u_{7}\right)\right]=0
$$

We get the eigenvalues of $J\left(E_{1}\right)$ in the $x_{3}, x_{4}$ direction respectively as :

$$
\left.\begin{array}{l}
\lambda_{x_{3}}=\frac{e u_{2}\left(1-m_{1}\right) \bar{x}_{1}+e u_{4}\left(1-m_{2}\right) \bar{x}_{2}}{u_{3}+\left(1-m_{1}\right) \bar{x}_{1}+\left(1-m_{2}\right) \bar{x}_{2}}-u_{7} \\
\lambda_{x_{4}}=u_{9}\left(1-m_{1}\right) \bar{x}_{1}-u_{10}
\end{array}\right\}
$$

Hence, we get the other two eigenvalues of $J\left(E_{1}\right)$ in the $x_{1}, x_{2}$ direction as:

$$
\lambda_{x_{1}}, \lambda_{x_{2}}=\frac{-B_{1}}{2} \pm \frac{1}{2} \sqrt{B_{1}^{2}-4 B_{2}}
$$

Then all the eigenvalues have negative real parts if the following conditions hold:

$\left.\begin{array}{c}u_{9}\left(1-m_{1}\right) \bar{x}_{1}<u_{10} \\ e u_{2}\left(1-m_{1}\right) \bar{x}_{1}+e u_{4}\left(1-m_{2}\right) \bar{x}_{2}<u_{7}\left(\begin{array}{c}u_{3}+\left(1-m_{1}\right) \bar{x}_{1} \\ +\left(1-m_{2}\right) \bar{x}_{2}\end{array}\right)\end{array}\right\}$

So, $E_{1}$ is a local stable in the $R_{+}^{4}$. And it is unstable point on the other hand.

Thus Jacobain matrix of system (2) at $E_{2}$ is a given by:

Where

$$
J\left(E_{2}\right)=\left[\begin{array}{cccc}
c_{11} & c_{12} & c_{13} & -\left(1-m_{1}\right) \hat{x}_{1} \\
c_{21} & c_{22} & c_{23} & 0 \\
c_{31} & c_{23} & 0 & -u_{6} \hat{x}_{3} \\
0 & 0 & 0 & u_{8} \hat{x}_{3}+u_{9}\left(1-m_{1}\right) \hat{x}_{1}-u_{10}
\end{array}\right]
$$

$$
\begin{aligned}
& c_{11}=-u_{1}-\left[\frac{u_{2} u_{3}\left(1-m_{1}\right) \hat{x}_{3}+u_{2}\left(1-m_{1}\right)\left(1-m_{2}\right) \hat{x}_{2} \hat{x}_{3}}{\left(u_{3}+\left(1-m_{1}\right) \hat{x}_{1}+\left(1-m_{2}\right) \hat{x}_{2}\right)^{2}}\right] \\
& c_{12} \\
& =1-2 \hat{x}_{2}+\left[\frac{u_{2}\left(1-m_{1}\right)\left(1-m_{2}\right) \hat{x}_{1} \hat{x}_{3}}{\left(u_{3}+\left(1-m_{1}\right) \hat{x}_{1}+\left(1-m_{2}\right) \hat{x}_{2}\right)^{2}}\right] \\
& \begin{array}{r}
c_{13}=\frac{-u_{2}\left(1-m_{1}\right) \hat{x}_{1}}{u_{3}+\left(1-m_{1}\right) \hat{x}_{1}+(1-} \\
=-\left(1-m_{1}\right) \hat{x}_{1} \\
c_{21}=u_{1}+\frac{u_{4}\left(1-m_{1}\right)\left(1-m_{2}\right) \hat{x}_{2} \hat{x}_{3}}{\left(u_{3}+\left(1-m_{1}\right) \hat{x}_{1}+\left(1-m_{2}\right) \hat{x}_{2}\right)^{2}}
\end{array} \\
& c_{22}=-u_{5}-\left[\frac{u_{3} u_{4}\left(1-m_{2}\right) \hat{x}_{3}+u_{4}\left(1-m_{1}\right)\left(1-m_{2}\right) \hat{x}_{1} \hat{x}_{3}}{\left(u_{3}+\left(1-m_{1}\right) \hat{x}_{1}+\left(1-m_{2}\right) \hat{x}_{2}\right)^{2}}\right]
\end{aligned}
$$




$$
\begin{aligned}
& c_{23} \\
& =\frac{-u_{4}\left(1-m_{2}\right) \hat{x}_{2}}{u_{3}+\left(1-m_{1}\right) \hat{x}_{1}+\left(1-m_{2}\right) \hat{x}_{2}} \\
& c_{31} \\
& =\frac{e u_{2} u_{3}\left(1-m_{1}\right) \hat{x}_{3}+\left[u_{2}-u_{4}\right] e\left(1-m_{1}\right)\left(1-m_{2}\right) \hat{x}_{2} \hat{x}_{3}}{\left(u_{3}+\left(1-m_{1}\right) \hat{x}_{1}+\left(1-m_{2}\right) \hat{x}_{2}\right)^{2}} \\
& c_{32} \\
& =\frac{e u_{3} u_{4}\left(1-m_{2}\right) \hat{x}_{3}+\left[u_{4}-u_{2}\right] e\left(1-m_{1}\right)\left(1-m_{2}\right) \hat{x}_{1} \hat{x}_{3}}{\left(u_{3}+\left(1-m_{1}\right) \hat{x}_{1}+\left(1-m_{2}\right) \hat{x}_{2}\right)^{2}} \\
& c_{33}=\frac{e u_{2}\left(1-m_{1}\right) \hat{x}_{1}+e u_{4}\left(1-m_{2}\right) \hat{x}_{2}}{u_{3}+\left(1-m_{1}\right) \hat{x}_{1}+\left(1-m_{2}\right) \hat{x}_{2}}-u_{7} \\
& =0
\end{aligned}
$$

Then the eigenvalues of $J\left(E_{2}\right)$ are

$$
\begin{aligned}
& \quad\left[\lambda-\left(u_{8} \hat{x}_{3}+u_{9}\left(1-m_{1}\right) \hat{x}_{1}-u_{10}\right]\left[\lambda^{3}+\hat{A}_{1} \lambda^{2}+\hat{A}_{2} \lambda+\hat{A}_{3}\right]=0\right. \\
& \quad \hat{A}_{1}=-\left[c_{11}+c_{22}+c_{33}\right] \\
& \hat{A}_{2}=c_{11} c_{22}-c_{12} c_{21}+c_{11} c_{33}-c_{13} c_{31}+c_{22} c_{33}-c_{23} c_{32} \\
& \hat{A}_{3}=c_{11} c_{22} c_{33}-c_{12} c_{23} c_{31}-c_{13} c_{21} c_{32}+c_{13} c_{22} c_{31}+c_{11} c_{23} c_{32}+c_{12} c_{21} c_{33} \\
& \text { Accordingly, either } \\
& \quad\left[\lambda-\left(u_{8} \hat{x}_{3}+u_{9}\left(1-m_{1}\right) \hat{x}_{1}-u_{10}\right]=0\right. \\
& \text { or } \\
& \lambda^{3}+\hat{A}_{1} \lambda^{2}+\hat{A}_{2} \lambda+\hat{A}_{3}=0
\end{aligned}
$$

Hence from equation (9b) we obtain

$$
\left.\lambda \hat{x}_{4}=u_{8} \hat{x}_{3}+u_{9}\left(1-m_{1}\right) \hat{x}_{1}-u_{10}\right\}
$$

Which is negative if the following condition hold

$$
u_{8} \hat{x}_{3}+u_{9}\left(1-m_{1}\right) \hat{x}_{1}<u_{10}
$$

Since $\widehat{\mathrm{A}}_{1}>0$, then by using Routh-Hurwitz criterion eq.(9c) has roots with negative real parts if $\widehat{\mathrm{A}}_{3}>0$ and

$\Delta=\hat{A}_{1} \hat{A}_{2}-\hat{A}_{3}=\left(c_{11}+c_{22}\right)\left(c_{12} c_{21}-c_{11} c_{22}\right)+c_{11} c_{13} c_{31}+c_{12} c_{23} c_{31}+\left(c_{22} c_{23}+c_{13} c_{21}\right) c_{32}$

Now, according to the form of $\widehat{\mathrm{A}}_{3}$ and signs of the jacobian matrix elements all terms of $\widehat{\mathrm{A}}_{3}$ are positive, while the first one will be positive ander the following conditions :

$$
\begin{aligned}
& \quad\left[u_{3}+\left(1-m_{1}\right) \hat{x}_{1}\right] u_{4}>u_{2}\left(1-m_{1}\right) \hat{x}_{1} \\
& {\left[u_{2}+\left(1-m_{2}\right) \hat{x}_{2}\right]>u_{4}\left(1-m_{2}\right) \hat{x}_{2}}
\end{aligned}
$$

However $\Delta$ becomes positives, since the first four terms of $\Delta$ are positive, while the last one will be positive if in addition to the condition $\mathrm{c}_{22} \mathrm{c}_{23}+\mathrm{c}_{13} \mathrm{c}_{21}>0$ the following condition holds :

$$
\begin{gathered}
{\left[u_{8} \hat{x}_{3}+u_{9}\left(1-m_{1}\right) \hat{x}_{1}\right]<u_{10}} \\
{\left[u_{3}+\left(1-m_{1}\right) \hat{x}_{1}\right] u_{4}>u_{8}\left(1-m_{1}\right) \hat{x}_{1}} \\
u_{2}\left[u_{3}+\left(1-m_{2}\right) \hat{x}_{2}\right]>u_{4}\left(1-m_{2}\right) \hat{x}_{2} \\
\hat{x}_{2}>\frac{1}{2}\left[1+\frac{u_{2}\left(1-m_{1}\right)\left(1-m_{2}\right) \hat{x}_{1} \hat{x}_{3}}{\left(u_{3}+\left(1-m_{1}\right) \hat{x}_{1}+\left(1-m_{2}\right) \hat{x}_{2}\right)^{2}}\right]
\end{gathered}
$$

Thus by Routh-Hurwitz criterion all the eigen values $J\left(E_{2}\right)$ have negative real parts so the steady state point $\mathrm{E}_{2}=\left(\hat{\mathrm{x}}_{1}, \hat{\mathrm{x}}_{2}, \hat{\mathrm{x}}_{3}, 0\right)$ is local stable.

The Jacobian matrix of system (2) at steady state point $E_{3}=\left(\breve{x}_{1}, \breve{x}_{2}, \breve{x}_{3}, \breve{x}_{4}\right)$ : 
Where

$$
J\left(E_{3}\right)=\left[\begin{array}{cccc}
d_{11} & d_{12} & d_{13} & -\left(1-m_{1}\right) \breve{x}_{1} \\
d_{21} & d_{22} & d_{23} & 0 \\
d_{31} & d_{23} & d_{33} & -u_{6} \breve{x}_{3} \\
u_{9}\left(1-m_{1}\right) \breve{x}_{4} & 0 & u_{8} \breve{x}_{4} & u_{8} \breve{x}_{3}+u_{9}\left(1-m_{1}\right) \breve{x}_{1}-u_{10}
\end{array}\right]
$$

$$
\begin{gathered}
d_{11}=-\left[u_{1}+\left(1-m_{1}\right) \breve{x}_{4}\right]-\left[\frac{u_{2} u_{3}\left(1-m_{1}\right) \breve{x}_{3}+u_{2}\left(1-m_{1}\right)\left(1-m_{2}\right) \breve{x}_{2} \breve{x}_{3}}{\left(u_{3}+\left(1-m_{1}\right) \breve{x}_{1}+\left(1-m_{2}\right) \breve{x}_{2}\right)^{2}}\right] \\
d_{12} \\
=1-2 \breve{x}_{2}+\left[\frac{u_{2}\left(1-m_{1}\right)\left(1-m_{2}\right) \breve{x}_{1} \breve{x}_{3}}{\left(u_{3}+\left(1-m_{1}\right) \breve{x}_{1}+\left(1-m_{2}\right) \breve{x}_{2}\right)^{2}}\right] \\
d_{13}=\frac{-u_{2}\left(1-m_{1}\right) \breve{x}_{1}}{u_{3}+\left(1-m_{1}\right) \breve{x}_{1}+\left(1-m_{2}\right) \breve{x}_{2}} \\
d_{21}=u_{1}+\frac{u_{4}\left(1-m_{1}\right)\left(1-m_{2}\right) \breve{x}_{2} \breve{x}_{3}}{\left(u_{3}+\left(1-m_{1}\right) \breve{x}_{1}+\left(1-m_{2}\right) \breve{x}_{2}\right)^{2}} \\
d_{22}=-u_{5}-\left[\frac{u_{3} u_{4}\left(1-m_{2}\right) \breve{x}_{3}+u_{4}\left(1-m_{1}\right)\left(1-m_{2}\right) \breve{x}_{1} \breve{x}_{3}}{\left(u_{3}+\left(1-m_{1}\right) \breve{x}_{1}+\left(1-m_{2}\right) \breve{x}_{2}\right)^{2}}\right] \\
d_{23}=\frac{-u_{4}\left(1-m_{2}\right) \breve{x}_{2}}{u_{3}+\left(1-m_{1}\right) \breve{x}_{1}+\left(1-m_{2}\right) \breve{x}_{2}} \\
d_{31}=\frac{e u_{2} u_{3}\left(1-m_{1}\right) \breve{x}_{3}+\left[u_{2}-u_{4}\right] e\left(1-m_{1}\right)\left(1-m_{2}\right) \breve{x}_{2} \breve{x}_{3}}{\left(u_{3}+\left(1-m_{1}\right) \breve{x}_{1}+\left(1-m_{2}\right) \breve{x}_{2}\right)^{2}} \\
d_{32}=\frac{e u_{3} u_{4}\left(1-m_{2}\right) \breve{x}_{3}+\left[u_{4}-u_{2}\right] e\left(1-m_{1}\right)\left(1-m_{2}\right) \breve{x}_{1} \breve{x}_{3}}{\left(u_{3}+\left(1-m_{1}\right) \breve{x}_{1}+\left(1-m_{2}\right) \breve{x}_{2}\right)^{2}} \\
d_{33}=\frac{e u_{2}\left(1-m_{1}\right) \breve{x}_{1}+e u_{4}\left(1-m_{2}\right) \breve{x}_{2}}{u_{3}+\left(1-m_{1}\right) \breve{x}_{1}+\left(1-m_{2}\right) \breve{x}_{2}}-\left(u_{6} \breve{x}_{4}+u_{7}\right)=0
\end{gathered}
$$

It is easy to verify that, the linearized system of system (2) can be written as:

$$
\frac{d R}{d t}=\frac{d s}{d t}=J\left(E_{3}\right) S
$$

Here

$$
R=\left(x_{1}, x_{2}, x_{3}, x_{4}\right)^{t} \text { and } S=\left(s_{1}, s_{2}, s_{3}, s_{4}\right)^{t}
$$

Where

$$
\begin{aligned}
& s_{1}=x_{1}-\breve{x}_{1}, \quad s_{2}=x_{2}-\breve{x}_{2} \\
& s_{3}=x_{3}-\breve{x}_{3}, \quad s_{4}=x_{4}-\breve{x}_{4}
\end{aligned}
$$

Now, consider the following positive define function

$$
L_{2}=\frac{a_{1}}{2} s_{1}^{2}+\frac{a_{2}}{2} s_{2}^{2}+\frac{a_{3}}{2 \breve{x}_{3}} s_{3}^{2}+\frac{a_{4}}{2 \breve{x}_{4}} s_{4}^{2}
$$

It is clearly that $\mathrm{L}_{2}: \mathrm{R}_{+}^{4} \rightarrow$ Rbecontinuously differentiable function, So that $\mathrm{L}_{2}\left(\breve{\mathrm{x}}_{1}, \breve{\mathrm{x}}_{2}, \breve{\mathrm{x}}_{3}, \breve{\mathrm{x}}_{4}\right)=0, \mathrm{~L}_{2}\left(\mathrm{x}_{1}, \mathrm{x}_{2}, \mathrm{x}_{3}, \mathrm{x}_{4}\right)>0$ otherwise so by differentiating $\mathrm{L}_{2}$ with respect to time $\mathrm{t}$, gives:

$$
\frac{d L_{2}}{d t}=a_{1} s_{1} \frac{d s_{1}}{d t}+a_{2} s_{2} \frac{d s_{2}}{d t}+\frac{a_{3}}{\breve{x}_{3}} s_{3} \frac{d s_{3}}{d t}+\frac{a_{4}}{\breve{x}_{4}} s_{4} \frac{d s_{4}}{d t}
$$

We get:

$$
\begin{aligned}
\frac{d L_{2}}{d t}=-\left[u_{1}+\right. & \left.\left(1-m_{1}\right) \breve{x}_{4}\right]+\left[u_{2} u_{3}\left(1-m_{1}\right) \breve{x}_{3}+u_{2}\left(1-m_{1}\right)\left(1-m_{2}\right) \breve{x}_{2} \breve{x}_{3}\right] s_{1}^{2} \\
& -\left[2 \breve{x}_{2}-1-\frac{u_{2}\left(1-m_{1}\right)\left(1-m_{2}\right) \breve{x}_{1} \breve{x}_{3}}{\left(u_{3}+\left(1-m_{1}\right) \breve{x}_{1}+\left(1-m_{2}\right) \breve{x}_{2}\right)^{2}}\right] \\
& -\left[u_{1}+\frac{u_{4}\left(1-m_{1}\right)\left(1-m_{2}\right) \breve{x}_{2} \breve{x}_{3}}{u_{3}+\left(1-m_{1}\right) \breve{x}_{1}+\left(1-m_{2}\right) \breve{x}_{2}}\right] s_{1} s_{2} \\
& -\left[u_{5}+\frac{u_{3} u_{4}\left(1-m_{2}\right) \breve{x}_{3}+u_{4}\left(1-m_{1}\right)\left(1-m_{2}\right) \breve{x}_{1} \breve{x}_{3}}{\left(u_{3}+\left(1-m_{1}\right) \breve{x}_{1}+\left(1-m_{2}\right) \breve{x}_{2}\right)^{2}}\right] s_{2}^{2}
\end{aligned}
$$




$$
\frac{d L_{2}}{d t}=-\left[q_{11} s_{1}^{2}+q_{12} s_{1} s_{2}+q_{22} s_{2}^{2}\right]
$$

Where

$$
\left.\begin{array}{l}
q_{11}=\left[u_{1}+\left(1-m_{1}\right) \breve{x}_{4}\right]+\left[\frac{u_{2} u_{3}\left(1-m_{1}\right) \breve{x}_{3}+u_{2}\left(1-m_{1}\right)\left(1-m_{2}\right) \breve{x}_{2} \breve{x}_{3}}{\left(u_{3}+\left(1-m_{1}\right) \breve{x}_{1}+\left(1-m_{2}\right) \breve{x}_{2}\right)^{2}}\right] \\
q_{12}=2 \breve{x}_{2}-\left[1+\frac{u_{2}\left(1-m_{1}\right)\left(1-m_{2}\right) \breve{x}_{1} \breve{x}_{3}}{\left(u_{3}+\left(1-m_{1}\right) \breve{x}_{1}+\left(1-m_{2}\right) \breve{x}_{2}\right)^{2}}\right]+u_{1}+\frac{u_{4}\left(1-m_{1}\right)\left(1-m_{2}\right) \breve{x}_{2} \breve{x}_{3}}{\left(u_{3}+\left(1-m_{1}\right) \breve{x}_{1}+\left(1-m_{2}\right) \breve{x}_{2}\right)^{2}} \\
q_{22}=u_{5}+\frac{u_{3} u_{4}\left(1-m_{2}\right) \breve{x}_{3}+u_{4}\left(1-m_{1}\right)\left(1-m_{2}\right) \breve{x}_{1} \breve{x}_{3}}{\left(u_{3}+\left(1-m_{1}\right) \breve{x}_{1}+\left(1-m_{2}\right) \breve{x}_{2}\right)^{2}}
\end{array}\right\}
$$

Now, it easy we have:

$\frac{d L_{2}}{d t}<-\left[\sqrt{q_{11}} s_{1}+\sqrt{q_{22}} s_{2}\right]^{2}$ if and only if

$\breve{x}_{2}$

$>\frac{1}{2}\left[1+u_{2}\right.$

$\left.+\frac{\left(u_{2} \breve{x}_{1}+u_{4} \breve{x}_{2}\right)\left(1-m_{1}\right)\left(1-m_{2}\right) \breve{x}_{3}}{\left(u_{3}+\left(1-m_{1}\right) \breve{x}_{1}+\left(1-m_{2}\right) \breve{x}_{2}\right)^{2}}\right]$

Therefore, $\frac{d L_{2}}{d t}$ is negative definite and hence $L_{2}$ is a Lyapunov faction with respect to $E_{3}$ in the sub region $\Omega_{2} . S_{0} E_{3}$ is asymptotically stable. Note that the faction $L_{2}$ is approaching to infant as any of its components to the same and its positive definite $R_{+}^{4}$,however its derivative is negative definite on the sub region $\Omega_{2}$ due to the given sufficient conditions. Therefore $E_{3}$ is a globally asymptotically stable with in $\Omega_{2}$.

Theorem (2): Assume that $E_{1}$ is local stable in $R_{+}^{4}$ if the following condition hold

$$
\begin{aligned}
& x_{1}>\bar{x}_{1}+e \\
& x_{2}>\bar{x}_{2}+e \\
& x_{1}>\bar{x}_{1}+u_{9}
\end{aligned}
$$

$$
u_{6}>u_{8}
$$

Then the steady state point $E_{1}$ is global stable.

Proof: let the following function

$$
v_{1}\left(x_{1}, x_{2}, x_{3}, x_{4}\right)=\frac{c_{1}}{2}\left(x_{1}-\bar{x}_{1}\right)^{2}+\frac{c_{2}}{2}\left(x_{2}-\bar{x}_{2}\right)^{2}+c_{3} x_{3}+c_{4} x_{4}
$$

It is easy to see that $v_{1}\left(x_{1}, x_{2}, x_{3}, x_{4}\right) \in C^{\prime}\left(R_{+}^{4}, R\right)$, in addition, $v_{1}\left(\bar{x}_{1}, \bar{x}_{2}, 0,0\right)=0$ while $\mathrm{v}_{1}\left(\mathrm{x}_{1}, \mathrm{x}_{2}, \mathrm{x}_{3}, \mathrm{x}_{4}\right)>0 \quad \forall\left(\mathrm{x}_{1}, \mathrm{x}_{2}, \mathrm{x}_{3}, \mathrm{x}_{4}\right) \in \mathrm{R}_{+}^{4}$ and $\left(\mathrm{x}_{1}, \mathrm{x}_{2}, \mathrm{x}_{3}, \mathrm{x}_{4}\right) \neq\left(\overline{\mathrm{x}}_{1}, \overline{\mathrm{x}}_{2}, 0,0\right)$.

Furthermore, by the derivative with time and simplifying we get that:

$$
\begin{aligned}
\frac{d v_{1}}{d t}=-c_{1} u_{1}( & \left.x_{1}-\bar{x}_{1}\right)^{2}-c_{1}\left(x_{1}-\bar{x}_{1}\right)\left(x_{2}-\bar{x}_{2}\right)\left(x_{2}+\bar{x}_{2}\right)+c_{1}\left(x_{1}-\bar{x}_{1}\right)\left(x_{2}-\bar{x}_{2}\right) \\
& +c_{2} u_{1}\left(x_{1}-\bar{x}_{1}\right)\left(x_{2}-\bar{x}_{2}\right)-c_{2} u_{5}\left(x_{2}-\bar{x}_{2}\right)^{2} \\
& -\frac{c_{1}\left(x_{1}-\bar{x}_{1}\right) u_{2}\left(1-m_{1}\right) x_{1} x_{3}}{u_{3}+\left(1-m_{1}\right) x_{1}+\left(1-m_{2}\right) x_{2}}-c_{1}\left(x_{1}-\bar{x}_{1}\right)\left(1-m_{1}\right) x_{1} x_{4} \\
& -\frac{c_{2} u_{4}\left(1-m_{2}\right)\left(x_{2}-\bar{x}_{2}\right) x_{2} x_{3}}{u_{3}+\left(1-m_{1}\right) x_{1}+\left(1-m_{2}\right) x_{2}}+\frac{c_{3} e u_{2}\left(1-m_{1}\right) x_{1} x_{3}}{u_{3}+\left(1-m_{1}\right) x_{1}+\left(1-m_{2}\right) x_{2}} \\
& +\frac{c_{3} e u_{4}\left(1-m_{2}\right) x_{2} x_{3}}{u_{3}+\left(1-m_{1}\right) x_{1}+\left(1-m_{2}\right) x_{2}}-c_{3} u_{6} x_{3} x_{4}-c_{3} u_{7} x_{3}+c_{4} u_{8} x_{3} x_{4} \\
& +c_{4}\left(1-m_{1}\right) u_{9} x_{1} x_{4}-c_{4} u_{10} x_{4} .
\end{aligned}
$$

And then substituting $c_{1}=c_{2}=c_{3}=c_{4}=1$ in the above equation we get : 


$$
\begin{aligned}
\frac{d v_{1}}{d t}=-\left[u _ { 1 } \left(x_{1}\right.\right. & \left.\left.-\bar{x}_{1}\right)^{2}-\left(x_{2}+\bar{x}_{2}+1+u_{1}\right)\left(x_{1}-\bar{x}_{1}\right)\left(x_{2}-\bar{x}_{2}\right)+u_{5}\left(x_{2}-\bar{x}_{2}\right)^{2}\right] \\
& -\left[x_{1}-\bar{x}_{1}-e\right] \frac{u_{2}\left(1-m_{1}\right) x_{1} x_{3}}{u_{3}+\left(1-m_{1}\right) x_{1}+\left(1-m_{2}\right) x_{2}} \\
& -\left[x_{2}-\bar{x}_{2}-e\right] \frac{u_{3}\left(1-m_{2}\right) x_{2} x_{3}}{u_{3}+\left(1-m_{1}\right) x_{1}+\left(1-m_{2}\right) x_{2}}-\left[x_{1}-\bar{x}_{1}-u_{9}\right]\left(1-m_{1}\right) x_{1} x_{4} \\
& -\left(u_{6}-u_{8}\right) x_{3} x_{4}-u_{7} x_{3}-u_{10} x_{4} .
\end{aligned}
$$

Obviously $\frac{d v_{1}}{d t}<0$ for every initial point and then $v_{1}$ is a Lya.function provided that conditions (11a11d) hold. Thus $E_{1}$ is global stable this completes the proof.

Theorem (3): Assume that $E_{2}=\left(\hat{x}_{1}, \hat{x}_{2}, \hat{x}_{3}, 0\right)$ is a locally in $R_{+}^{4}$, then it is global stable provided that the following conditions:

$$
\begin{aligned}
& {\left[1-\left(x_{2}+\hat{x}_{2}\right)+\frac{u_{2}\left(1-m_{1}\right)\left(1-m_{2}\right) x_{1} x_{3}}{k}+u_{1}+\frac{u_{4}\left(1-m_{1}\right)\left(1-m_{2}\right) \hat{x}_{2} \hat{x}_{3}}{k}\right]^{2}} \\
& <4\left(\frac{u_{1} k+u_{2}\left(1-m_{1}\right) \hat{x}_{3}}{k}\left[1-\left(1-m_{1}\right) \hat{x}_{1}\right]\right)\left(u_{5}\right. \\
& \left.+\frac{u_{4}\left(1-m_{2}\right) \hat{x}_{3}}{k}\left[1-\left(1-m_{2}\right) \hat{x}_{2}\right]\right) \\
& 1>\max \left\{\left(1-m_{1}\right) \hat{x}_{1},\left(1-m_{2}\right) \hat{x}_{2}\right\} \\
& u_{4}\left(1-m_{2}\right) \hat{x}_{2}>u_{2}\left(u_{3}+\left(1-m_{2}\right) \hat{x}_{2}\right. \\
& u_{2}\left(1-m_{1}\right) \hat{x}_{1}>u_{4}\left(u_{3}+\left(1-m_{1}\right) \hat{x}_{1}\right. \\
& \left(1-m_{1}\right) x_{1}^{2} u_{4}+u_{6} x_{3} x_{4}>\left(1-m_{1}\right) \hat{x}_{1} x_{1} x_{4}+u_{6} x_{4} \hat{x}_{3}
\end{aligned}
$$

Proof: Consider the following function

$$
v_{2}\left(x_{1}, x_{2}, x_{3}, x_{4}\right)=\frac{1}{2}\left(x_{1}-\hat{x}_{1}\right)^{2}+\frac{1}{2}\left(x_{2}-\hat{x}_{2}\right)^{2}+\left(x_{3}-\hat{x}_{3}-\hat{x}_{3} \ln \frac{x_{3}}{\hat{x}_{3}}\right)+x_{4}
$$

It is easy to see that

$\mathrm{v}_{2}\left(\mathrm{x}_{1}, \mathrm{x}_{2}, \mathrm{x}_{3}, \mathrm{x}_{4}\right) \in \mathrm{C}^{\prime}\left(\mathrm{R}_{+}^{4}, \mathrm{R}\right), \quad \forall\left(\mathrm{x}_{1}, \mathrm{x}_{2}, \mathrm{x}_{3}, \mathrm{x}_{4}\right) \in \mathrm{R}_{+}^{4}$ and $\left(\mathrm{x}_{1}, \mathrm{x}_{2}, \mathrm{x}_{3}, \mathrm{x}_{4}\right) \neq\left(\hat{\mathrm{x}}_{1}, \hat{\mathrm{x}}_{2}, \hat{\mathrm{x}}_{3}, 0\right)$

Furthermore by taking the derivative with time and simplifying we get that:

$$
\begin{aligned}
& \frac{d v_{2}}{d t} \\
& =\left(x_{1}-\hat{x}_{1}\right)\left[\left(x_{2}-\hat{x}_{2}\right)-\left(x_{2}^{2}-\hat{x}_{2}^{2}\right)-u_{1}\left(x_{1}-\hat{x}_{1}\right)\right. \\
& \left.-u_{2}\left(1-m_{1}\right)\left(\frac{x_{1} x_{3}\left(u_{3}+\left(1-m_{1}\right) \hat{x}_{1}+\left(1-m_{2}\right) \hat{x}_{2}\right)-\hat{x}_{1} \hat{x}_{3}\left(u_{3}+\left(1-m_{1}\right) x_{1}+\left(1-m_{2}\right) x_{2}\right)}{\left(u_{3}+\left(1-m_{1}\right) x_{1}+\left(1-m_{2}\right) x_{2}\right)\left(u_{3}+\left(1-m_{1}\right) \hat{x}_{1}+\left(1-m_{2}\right) \hat{x}_{2}\right)}\right)\right] \\
& +\left(x_{2}-\hat{x}_{2}\right)\left[u_{1}\left(x_{1}-\hat{x}_{1}\right)-u_{5}\left(x_{2}-\hat{x}_{2}\right)\right. \\
& \left.-u_{4}\left(1-m_{2}\right)\left(\frac{x_{2} x_{3}\left(u_{3}+\left(1-m_{1}\right) \hat{x}_{1}+\left(1-m_{2}\right) \hat{x}_{2}\right)-\hat{x}_{2} \hat{x}_{3}\left(u_{3}+\left(1-m_{1}\right) x_{1}+\left(1-m_{2}\right) x_{2}\right)}{\left(u_{3}+\left(1-m_{1}\right) x_{1}+\left(1-m_{2}\right) x_{2}\right)\left(u_{3}+\left(1-m_{1}\right) \hat{x}_{1}+\left(1-m_{2}\right) \hat{x}_{2}\right)}\right)\right] \\
& +\left(x_{3}\right. \\
& \left.-\hat{x}_{3}\right)\left[\left(\frac{e u_{2}\left(1-m_{1}\right)\left(x_{1}\left(u_{3}+\left(1-m_{1}\right) \hat{x}_{1}+\left(1-m_{2}\right) \hat{x}_{2}\right)-\hat{x}_{1}\left(u_{3}+\left(1-m_{1}\right) x_{1}+\left(1-m_{2}\right) x_{2}\right)\right.}{\left(u_{3}+\left(1-m_{1}\right) x_{1}+\left(1-m_{2}\right) x_{2}\right)\left(u_{3}+\left(1-m_{1}\right) \hat{x}_{1}+\left(1-m_{2}\right) \hat{x}_{2}\right)}\right)\right. \\
& +e u_{4}\left(1-m_{2}\right)\left(\frac{\left(x_{2}\left(u_{3}+\left(1-m_{1}\right) \hat{x}_{1}+\left(1-m_{2}\right) \hat{x}_{2}\right)-\hat{x}_{2}\left(u_{3}+\left(1-m_{1}\right) x_{1}+\left(1-m_{2}\right) x_{2}\right)\right.}{\left(u_{3}+\left(1-m_{1}\right) x_{1}+\left(1-m_{2}\right) x_{2}\right)\left(u_{3}+\left(1-m_{1}\right) \hat{x}_{1}+\left(1-m_{2}\right) \hat{x}_{2}\right)}\right) \\
& \left.-u_{6} x_{4}\right]+\left[u_{8} x_{3} x_{4}+\left(1-m_{1}\right) u_{9} x_{1} x_{4}-u_{10} x_{4}\right]
\end{aligned}
$$


Obviously $\frac{\mathrm{dv}_{2}}{\mathrm{dt}}<0$ for every initial point and then $\mathrm{v}_{2}$ is a Lyap. function provided that conditions (11a-11f) hold. Thus $E_{2}$ is global stable this completes the proof.

Theorem (4): Assume that $E_{3}$ is local stable in $R_{+}^{4}$. Then, it is a global stable in sub region of $R_{+}^{4}$ that satisfies the following conditions

$$
\begin{gathered}
x_{2}+x_{2}^{*}>1+u_{1} \\
k_{1}+k_{2}>u_{2}\left(1-m_{1}\right)\left(x_{1}-x_{1}^{*}\right) k_{3} \\
\quad+u_{4}\left(1-m_{2}\right)\left(x_{2}-x_{2}^{*}\right) k_{4} \\
{\left[u_{2}+u_{4}\right] e\left(1-m_{1}\right)\left(1-m_{2}\right) x_{1}^{*} x_{2}\left(x_{3}-x_{3}^{*}\right)} \\
\quad>e\left(x_{3}-x_{3}^{*}\right)\left[u_{2}\left(1-m_{1}\right) k_{5}+u_{4}\left(1-m_{2}\right) k_{6}\right.
\end{gathered}
$$

Where

$$
\begin{gathered}
k_{1}=u_{2}\left(1-m_{1}\right) x_{1} x_{3}\left(x_{1}-x_{1}^{*}\right) \\
k_{2}=u_{4}\left(1-m_{2}\right) x_{2} x_{3}\left(x_{2}-x_{2}^{*}\right) \\
k_{3}=\left(1+\left(1-m_{1}\right)\left(x_{1}-x_{1}^{*}\right)\right) x_{1}^{*} x_{3}^{*}+\left(1-m_{2}\right) x_{1}^{*} x_{2}^{*}\left(x_{2}-x_{2}^{*}\right) \\
k_{4}=\left[1+\left(1-m_{1}\right)\left(x_{1}-x_{1}^{*}\right)+\left(1-m_{2}\right)\left(x_{2}-x_{2}^{*}\right)\right] x_{2}^{*} x_{3}^{*} \\
k_{5}=u_{3}\left(x_{1}-x_{1}^{*}\right)+\left(1-m_{2}\right) x_{1} x_{2}^{*} \\
k_{6}=u_{3}\left(x_{2}-x_{2}^{*}\right)+
\end{gathered}
$$

Proof: consider the following function

$v_{3}\left(x_{1}, x_{2}, x_{3}, x_{4}\right)=\frac{1}{2}\left(x_{1}-x_{1}^{*}\right)^{2}+\frac{1}{2}\left(x_{2}-x_{2}^{*}\right)^{2}+\left(x_{3}-x_{3}^{*}-x_{3}^{*} \ln \frac{x_{3}}{x_{3}^{*}}\right)+\left(x_{4}-x_{4}^{*}-x_{4}^{*} \ln \frac{x_{4}}{x_{4}^{*}}\right)$

It is easy to verify that $v_{3}\left(x_{1}, x_{2}, x_{3}, x_{4}\right) \in C^{\prime}\left(R_{+}^{4}, R\right) \operatorname{andv}_{3}\left(x_{1}^{*}, x_{2}^{*}, x_{3}^{*}, x_{4}^{*}\right)=0$ while $\mathrm{v}_{3}\left(\mathrm{x}_{1}, \mathrm{x}_{2}, \mathrm{x}_{3}, \mathrm{x}_{4}\right)>0$ for all $\left(\mathrm{x}_{1}, \mathrm{x}_{2}, \mathrm{x}_{3}, \mathrm{x}_{4}\right) \in \mathrm{R}_{+}^{4}$ and $\left(\mathrm{x}_{1}, \mathrm{x}_{2}, \mathrm{x}_{3}, \mathrm{x}_{4}\right) \neq\left(\mathrm{x}_{1}^{*}, \mathrm{x}_{2}^{*}, \mathrm{x}_{3}^{*}, \mathrm{x}_{4}^{*}\right)$ then by find the derivative with time, also simplifying it we get:

$$
\begin{aligned}
& \frac{d v_{3}}{d t} \\
& =\left(x_{1}-\breve{x}_{1}\right)\left[\left(x_{2}-\breve{x}_{2}\right)-\left(x_{2}^{2}-\breve{x}_{2}^{2}\right)-u_{1}\left(x_{1}-\breve{x}_{1}\right)-\left(1-m_{1}\right)\left(x_{1} x_{4}-\breve{x}_{1} \breve{x}_{4}\right)\right. \\
& \left.-u_{2}\left(1-m_{1}\right)\left(\frac{x_{1} x_{3}\left(u_{3}+\left(1-m_{1}\right) \breve{x}_{1}+\left(1-m_{2}\right) \breve{x}_{2}\right)-\breve{x}_{1} \breve{x}_{3}}{\left(u_{3}+\left(1-m_{1}\right) x_{1}+\left(1-m_{2}\right) x_{2}\right)\left(u_{3}+\left(1-m_{1}\right) \breve{x}_{1}+\left(1-m_{2}\right) \breve{x}_{2}\right)}\right)\right] \\
& +\left(x_{2}-\breve{x}_{2}\right)\left[u_{1}\left(x_{1}-\breve{x}_{1}\right)-u_{5}\left(x_{2}-\breve{x}_{2}\right)\right. \\
& \left.-u_{4}\left(1-m_{2}\right)\left(\frac{x_{2} x_{3}\left(u_{3}+\left(1-m_{1}\right) \breve{x}_{1}+\left(1-m_{2}\right) \breve{x}_{2}\right)-\breve{x}_{1} \breve{x}_{3}\left(u_{3}+\left(1-m_{1}\right) x_{1}+\left(1-m_{2}\right) x_{2}\right)}{\left(u_{3}+\left(1-m_{1}\right) x_{1}+\left(1-m_{2}\right) x_{2}\right)\left(u_{3}+\left(1-m_{1}\right) \breve{x}_{1}+\left(1-m_{2}\right) \breve{x}_{2}\right)}\right)\right] \\
& +\left(x_{3}\right. \\
& \left.-\breve{x}_{3}\right)\left[\left(\frac{e u_{2}\left(1-m_{1}\right)\left(x_{1}\left(u_{3}+\left(1-m_{1}\right) \breve{x}_{1}+\left(1-m_{2}\right) \breve{x}_{2}\right)-\breve{x}_{1}\left(u_{3}+\left(1-m_{1}\right) x_{1}+\left(1-m_{2}\right) x_{2}\right)\right.}{\left(u_{3}+\left(1-m_{1}\right) x_{1}+\left(1-m_{2}\right) x_{2}\right)\left(u_{3}+\left(1-m_{1}\right) \breve{x}_{1}+\left(1-m_{2}\right) \breve{x}_{2}\right)}\right)\right. \\
& +e u_{4}(1 \\
& \left.-m_{2}\right)\left(\frac{\left(x_{2}\left(u_{3}+\left(1-m_{1}\right) \breve{x}_{1}+\left(1-m_{2}\right) \breve{x}_{2}\right)-\breve{x}_{2}\left(u_{3}+\left(1-m_{1}\right) x_{1}+\left(1-m_{2}\right) x_{2}\right)\right.}{\left(u_{3}+\left(1-m_{1}\right) x_{1}+\left(1-m_{2}\right) x_{2}\right)\left(u_{3}+\left(1-m_{1}\right) \breve{x}_{1}+\left(1-m_{2}\right) \breve{x}_{2}\right)}\right) u_{6}\left(x_{4}\right. \\
& \left.\left.-\breve{x}_{4}\right)\right]+\left(x_{4}-\breve{x}_{4}\right)\left[u_{8}\left(x_{3}-\breve{x}_{3}\right)+\left(1-m_{1}\right) u_{9}\left(x_{1}-\breve{x}_{1}\right)\right] .
\end{aligned}
$$

Clearly, $\frac{d v_{3}}{d t}<0$, and then $v_{3}$ is a Lyap. function provided that the given conditions (13a-13c) hold. Therefore, $E_{3}$ is global stable in the interior of a basin of attraction of $E_{3}$ and the proof is complete.

\section{Numerical illustrate}

In this section, the dynamical behavior of system (2) is studied numerically for different ets of initial values and different sets of parameters values. 
It is observed that for the following set of hypothetical parameters system (2) has an asymptotical stable steady state point $\mathrm{E}_{1}=(0.2,0.99,0.0)$ as shown in Figure-1

$\mathrm{u}_{1}=0.05, \mathrm{u}_{2}=0.00001, \mathrm{u}_{3}=0.25, \mathrm{u}_{4}=0.00001, \mathrm{u}_{5}=0.01$

$\mathrm{u}_{6}=0.1, \mathrm{u}_{7}=0.05, \mathrm{u}_{8}=0.002 ; \mathrm{u}_{9}=0.02, \mathrm{u}_{10}=0.1$

$\mathrm{m}_{1}=0.01, \mathrm{~m}_{2}=0.02, \mathrm{e}=0.003$
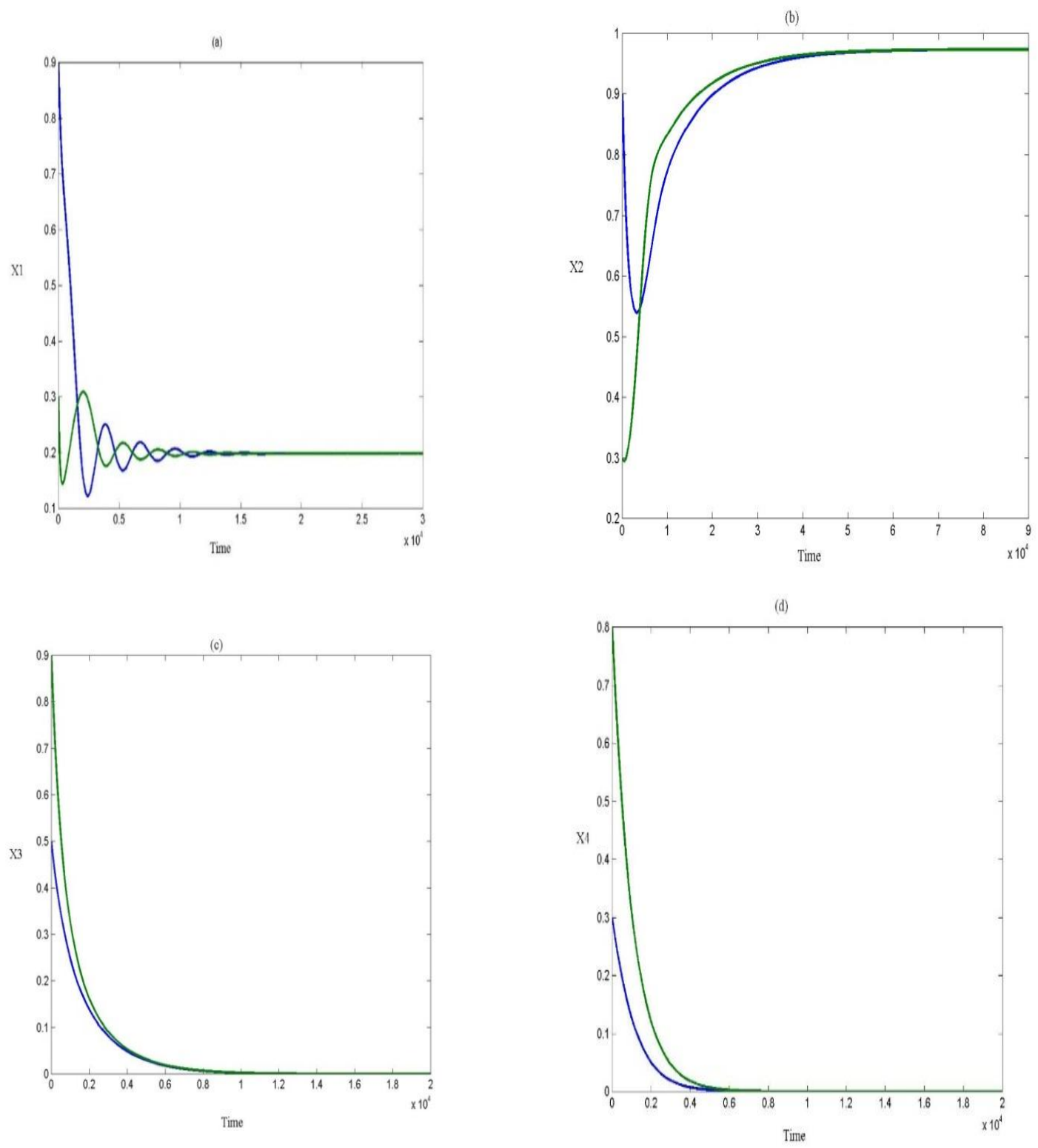

Figure 1-Trajectory of system (2) that begin from different initial point, $(0.9,0.7,0.5,0.3)$ and $(0.3,0.9,0.9,0.8)$ for the data given by Eq. (14). (a) Trajectories of immature prey as a function of time (b) Trajectory of mature prey as a function of time. (c) Trajectory of susceptible predator as a function of a function of time.

from values of parameters that given in Eq. (14) with $\mathrm{u}_{6}=0.001, \mathrm{u}_{9}=0.0002$.the solution of system (2) approaches to $\mathrm{E}_{2}=(0.5,0.9,0.6,0)$ as shown in Figure- 2 

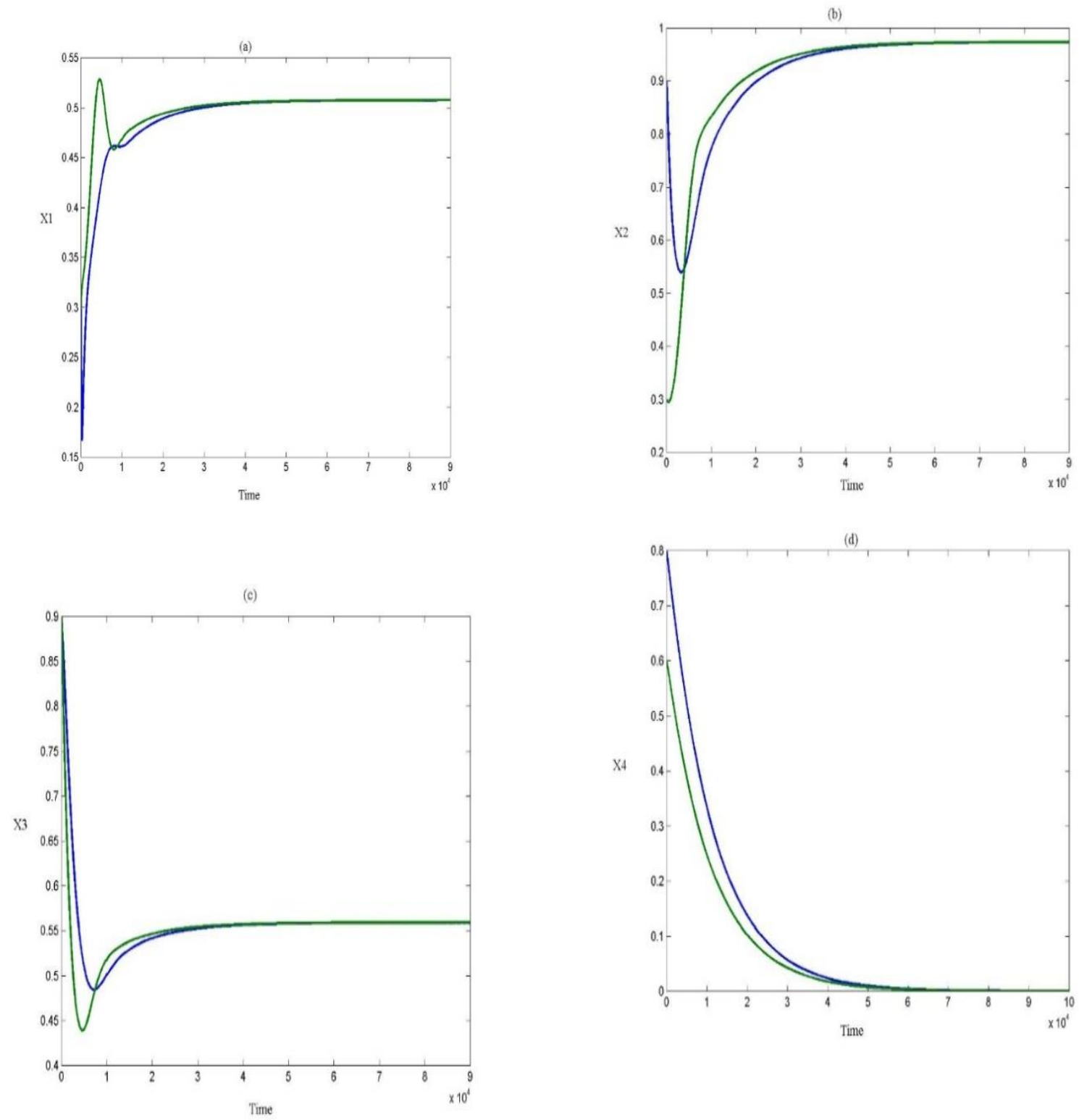

Figure 2-Trajectory of solution of system (2) for above parameters from different set of initial points $(0.9,0.7,0.5,0.3)$ and $(0.3,0.3,0.9,0.6)$. (a) Trajectories of immature prey (b) Trajectory of mature prey (c) Trajectory of susceptible predator (d) Trajectory of infected predator.

It is observed that for the following set of hypothetical parameters that satisfies stable conditions of positive steady state point $\mathrm{E}_{3}=(0.4,0.8,0.5,0.3)$ system (2) has asymptotic stable positive steady state point as shown in Figure-3

$u_{1}=0.05, u_{2}=0.00001 u_{3}=0.25, u_{4}=0.05, u_{5}=0.01$
$\mathrm{u}_{6}=0.003, \mathrm{u}_{7}=0.05, \mathrm{u}_{8}=0.002, \mathrm{u}_{9}=0.02, \mathrm{u}_{10}=0.01$
$m_{1}=0.01, m_{2}=0.02, e=0.003$. 

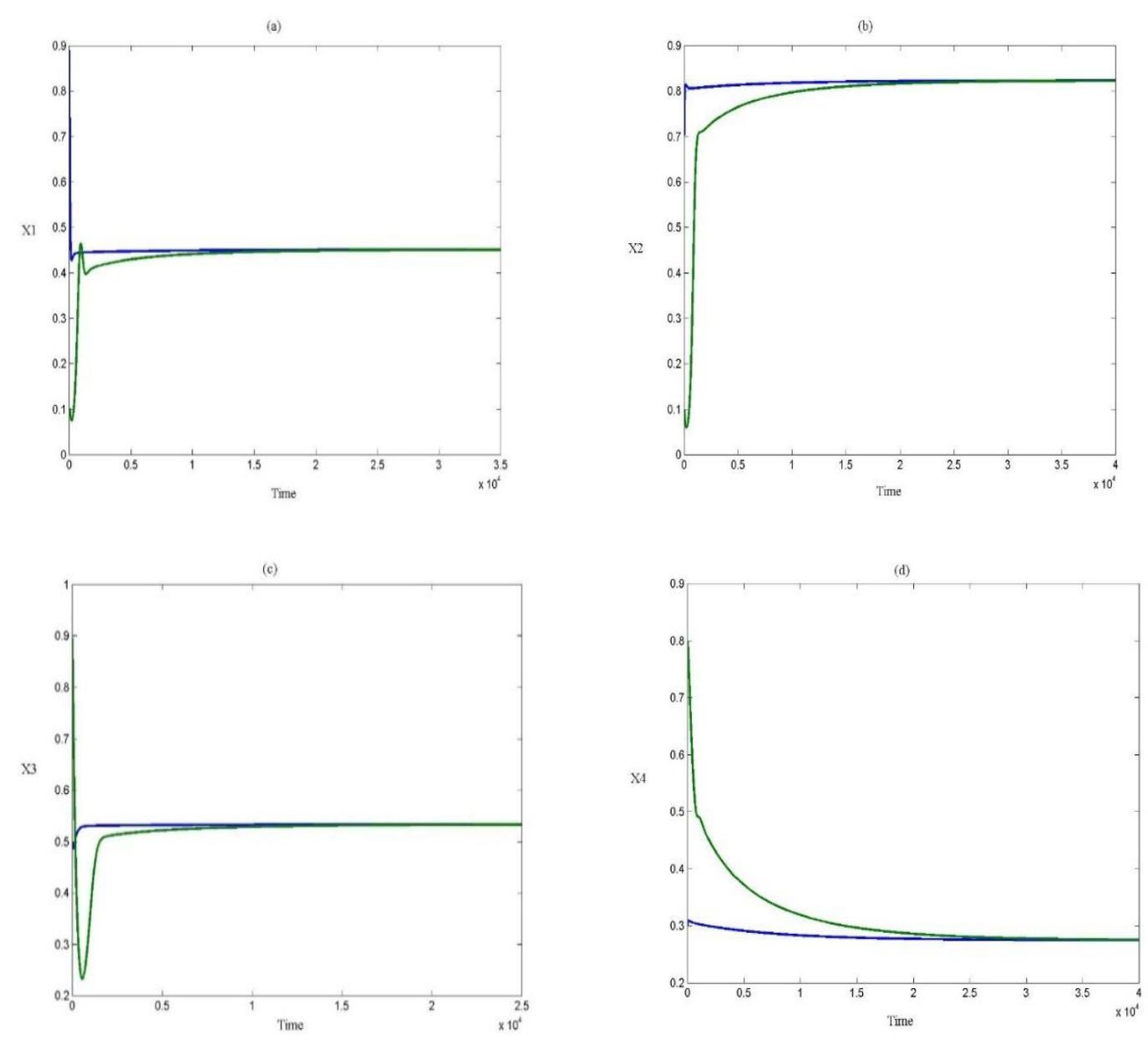

Figure 3-Trajectory of system (2) that begin from different initial points $(0.9,0.7,0.5,0.3)$ and $(0.1$, $0.1,0.9,0.8$ ). For the data given by $\operatorname{Eq}(15)$ (a) Trajectories of immature prey as a function of a time. (b) Trajectory of mature prey as a function of a time (c) Trajectory of susceptible predator as a function of a time (d) Trajectory of infected predator as a function of a time.

\section{References}

1. Anderson, R.M. and May, R.M. 1986. The invasion and spread ofinfectious disease with in Animal and plant communities, Philos. Trans. R. Soc. Lond. Biol. Sci. 314: 533-570.

2. Haque, M., Zhen, J. and Venturino, E. 2009. Rich dynamics of LotkaVolterra type predator-prey model system with viral disease in prey species. Mathematical Methods in the Applied Sciences, 32: 875898 .

3. Xiao, Y. and Chen, L. Modeling and analysis of a predator-preymodel with disease in the prey. Mathematical Bioscences, 171: 59-82.

4. Kar, T.K., Ghorai, A. and Jana, S. 2012. Dynamics of pest and its predator model with disease in the pest and optimal use of pesticide. Journal of Theoretical Biology, 310(7): 187-198.

5. Hassan F., Ridha and Ahmed A. 2017. The Dynamical Behaviors for an Epidemic Disease Model with General Recovery Function, Sci.Int.(Lahore), 29(5: 007-1014.

6. Bairagi. N., Chaudhuri, S. and Chattopadhyay, J. 2009. Harvesting as a diseasecontrol measure in an eco-epidemiological system - Atheoretical study, Mathematical Biosciences, 217: 134-144.

7. Chakraborty, S., Pal, S. and Bairagi, N. 2010. Dynamics of a ratio-dependent eco-epidemiological system with prey harvesting, Nonlinear Analysis: Real World Applications, 11: 1862-1877.

8. Upadhyay, R.K. and Roy, P. 2014. Spread of a disease and its effect on population dynamics in an eco-epidemiological system, Communications in Nonlinear Science Numerical Simulation, 19(41): 70-4184.

9. Upadhyay, R.K., Bairagi, N. 2008. Kundu, K. J. Chattopadhyay, Chaos in ecoepidemiological problem of the Salton Sea and its possible control, Applied Mathematics and Computation, 196: $392-401$. 